\title{
Petaloid Monocotyledonous Flora of Tunceli Province (Turkey)
}

\section{Tunceli (Türkiye)'nin Monokotil Petaloidleri}

\author{
Research Article
}

Metin Armağan*

Department of Medicinal and Aromatic Plants, Buharkent Vocational School, Adnan Menderes University, Aydın, Turkey.

\section{A B S TR ACT}

\begin{abstract}
This study was carried out to determine the monocotyl petaloids of Tunceli Province (Turkey). The research area is located in B7 square and a little part in B8 square based on the quadratic system of Davis. In this research, the field studies were carried out in Tunceli during 2014-2015. Totally 150 taxa (142 species, 4 subspecies and 4 varieties) which are 33 endemic were identified belonging to 11 families. Of the taxa found in the area, 26 were newly recorded for the province of Tunceli. This study is part of the ongoing research to determine for Flora of Tunceli.
\end{abstract}

\section{Key Words}

Tunceli, petaloid, monocotyledonous, geophyte, Turkey.

\section{öz}

u çalışma, Tunceli (Türkiye) ilinin monokotil petaloidlerini tespit etmek amacıyla yapılmıştır. Araştırma alanı Davis'in kareleme sistemine göre çoğunluğu B7, az bir kısmı ise B8 karesindedir. Bu araştırmada, 2014-2015 yılları arasında Tunceli'de arazi çalışmaları yapılmıştır. Tayin edilen bitkilerden 11 familyaya ait toplam 150 takson (142 tür, 4 alttür ve 4 varyete) tespit edilmiş olup, bunlardan 33 tanesi endemiktir. Alanda tespit edilen taksonlardan 26 tanesi Tunceli ili için yeni kayıttır. Bu çalışma devam eden Tunceli Florası'nın belirlenmesi çalışmasının bir bölümüdür.

\section{Anahtar Kelimeler}

Tunceli, petaloid, monokotil, geofit, Türkiye.

Article History: Received: Feb 25, 2018; Revised: Mar 03, 2018; Accepted: Mar 05, 2018; Available Online: Mar $26,2018$. DOI: 10.15671/HJBC.2018.229

Correspondence to: M. Armağan, Dept. of Med. and Aromatic Plants, Buharkent Voc. School, Adnan Menderes Uni., Aydın, Turkey. 


\section{INTRODUCTION}

Turkey appears as a bridge between Europe and Asia. The flora of Turkey has rich and interesting plant diversity due to the interaction between Mediterranean, Irano-Turanian and Europe-Siberian phytogeographic regions [1]. Anatolia's location at the connections of Europe, the Middle East, Central Asia, and Africa and also its high topographic and climatic heterogeneities lead to high biodiversity and rate of endemism [2].
Tunceli is located on Anatolian diagonal in Eastern region of Turkey. Geography of Tunceli composes of significant elevations (Sultanbaba, Munzur, Hel-Yel-Zel, Buyerbaba, and Düzgünbaba etc.), the rivers (Munzur, Pülümür, Mercan, Tahar, and Perisuyu) and the valleys (Munzur, Pülümür, Rabat, Tahar, and Mercan etc.). The least area of agriculturally fit land of Eastern Anatolia is found in Tunceli, being 114.000 ha. Livestock raising is thus very important. Most of the grasslands are mowed for winter fodder [3].
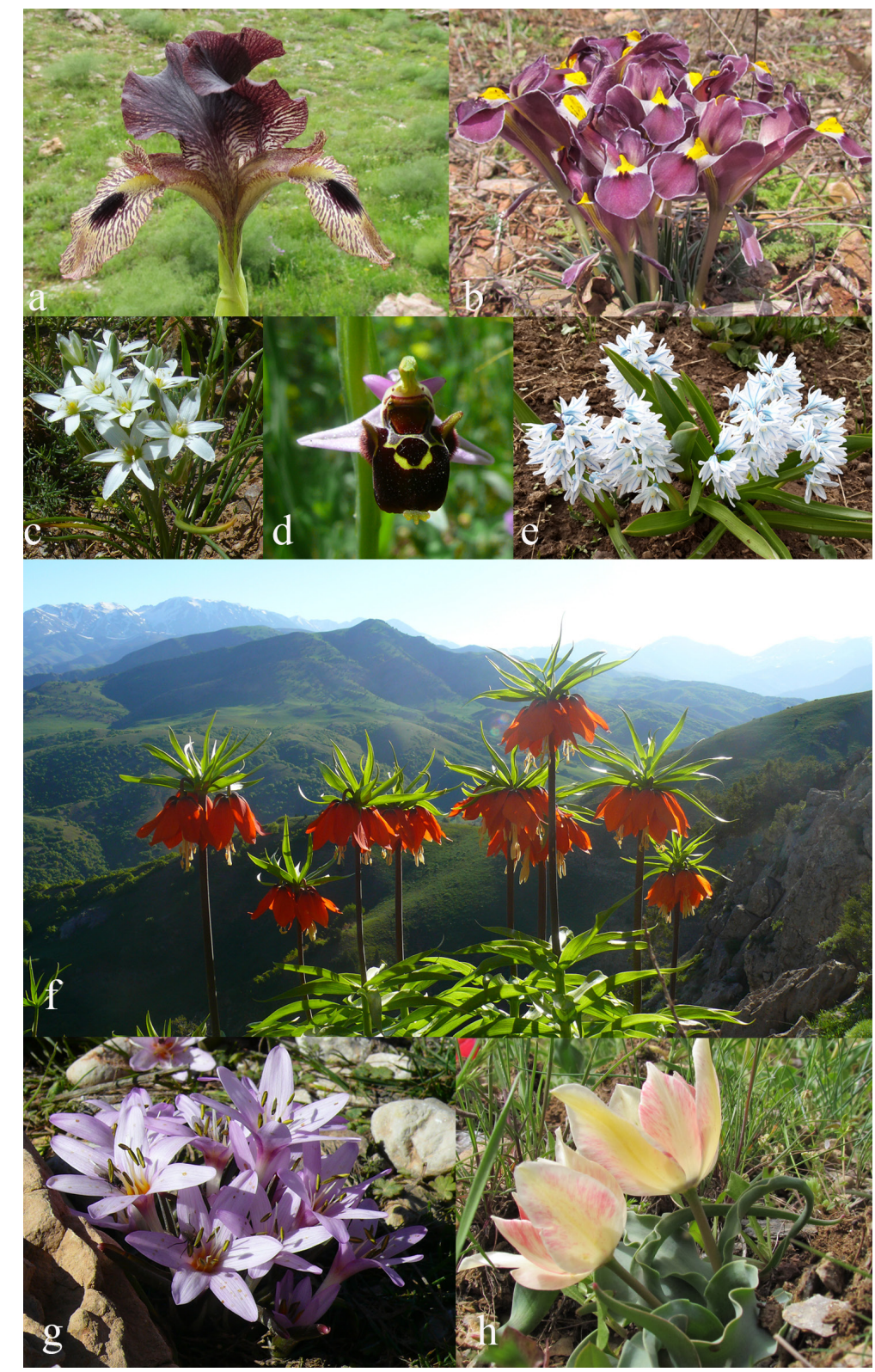

Figure 1. Some geophytes from Tunceli. a. Iris sari Schott ex Baker (endemic), b. Iris galatica Siehe (endemic), c. Ornithogalum munzurense Speta (endemic), d. Ophrys oestrifera M.Bieb., e. Puschkinia scilloides Adams, f. Fritillaria imperialis L. and Munzur mountains, g. Colchicum munzurense K.Perss. (endemic), h. Tulipa armena Boiss. 
The Munzur Mountains is the most famous and biggest mountains in Tunceli. The 1407 vascular plant taxa belonging to 98 families and 479 genera are reported in these mountains, which forming the border of Erzincan and Tunceli [4].

The geophytes -including some endemics- have been gathered unconsciously or consciously as ornamental, food or medicinal plants by the public collectors due to their economic value in the world ( $\mathrm{Fi}$ gure 1). Moreover, the deterioration of natural areas due to human influences threat to their generations. Therefore the conservation of plants species and the prepared reports about distributing areas are important for the biodiversity [5].

\section{MATERIALS and METHODS}

In this study, about 3400 plant samples were collected within the borders of Tunceli province during the vegetation seasons in 2014 and 2015, and photos, GPS coordinates, and habitat information were taken in their natural habitats. The collected samples were dried based to herbarium methods, and the collector numbers were given. Prepared samples with respect to herbarium techniques were stored in the AYDN and VANF herbaria. In order to compile an up-todate and inclusive list, the taxa identified in the study and in previous studies were given together in single list.

During the identification of samples, Flora of Turkey and the East Aegean Islands $[6,7,8]$ ) were initially used for the nomenclaturel checking of plant names it was mainly utilized from "Bizim Bitkiler [9]" and "The Plant List [10]". For the locality information of plants, the location, altitude, habitat, date of collection, number of collector, and the reference literature code are given respectively. Locality records belonging to the references were given unchanged in their original form. Tunceli name was not repeatedly written at the beginning of each locality because the study was carried out only in the Tunceli province. The phytogeographic region elements and the endemism status of the taxa were specified at the end of the record of localities.

Literatures and abbreviations used in the Floristic List as follow;

MA = This study, R4 = Reference 4, R6 = Reference 6, R8 = Reference 8, R11 = Reference 11, R12 =
Reference 12, R13 = Reference 13, R14 = Reference 14, R15 = Reference 15.

End. = Endemic, Ir.-Tur. = Irano - Turanian, Medit. = Mediterranean, Hyr. -Eux. = Hyrcano-Euxine, Eux. = Euxine, Euro.-Sib. $=$ Euro - Siberian, E.Medit. $=$ East Mediterranean.

AYDN $=$ Herbarium of Adnan Menderes University

VANF $=$ Herbarium of Yüzüncü Yıl University

\section{RESULTS}

Totally 150 taxa (142 species, 4 subspecies and 4 varieties) which are 33 endemic belonging to 11 families were identified. Of the taxa found in the area, 26 were newly recorded for the province of Tunceli. The list of taxa and the collection data were given below alphabetically order of the family, genus, species, and if it is present infraspecific category.

\section{ALISMATACEAE}

\section{Alisma lanceolatum With./Kurbağakaşığı}

Mazgirt, c. 3.3 km EESE Mazgirt, Yukarıoyumca, $1430 \mathrm{~m}$, boggy area besides lake, 11.vi.2013, V.Y.E. \& M. 13-0017 (R14).

\section{AMARYLLIDACEAE}

Allium akaka S.G.Gmel. ex Schult. \& Schult.f./Yer soğanı

Pülümür, $500 \mathrm{~m} \mathrm{~N}$ of Balpayam (3- $4 \mathrm{~km} \mathrm{~S}$ of Kırklar), 2400 m, mountain steppe, 18.vi.2014, Armağan 4844 (MA). Ir.-Tur.

\section{Allium ampeloprasum L./Pırasa}

Çemişgezek, between Bozağaç and Ulukale, $1 \mathrm{~km}$ E of Ulukale, $1080 \mathrm{~m}$, dried river bed, shade area, 28.vi.2014, Armağan 5148 (MA). Medit.

\section{Allium armenum Boiss. \& Kotschy/Pembesırım}

Pülümür, 2 km from Turnadere to Közlüce, 1575 m, rocky areas, 18.vi.2014, Armağan 4765 (MA); Ovacık, banks of Munzur Suyu, 1200 m, 18.vi.1979, Yıldırımlı 2034 (R4); Ovacık, Akyayık Köyü, 1315 m, 10.iv.2013, E.Yüce 2794 (R13); Munzur Vadisi (R15). Ir.-Tur., End.

\section{Allium asperiflorum Miscz./Benekli soğan}


Mazgirt, Akpazar town, $\mathrm{S}$ of Yenice (S of Şevki Mezrası), 1270 m, steppe, 02.vi.2014, Armağan 4129 (MA). Ir.-Tur., End.

\section{Allium atroviolaceum Boiss./Liflikörmen}

Mazgirt, $1 \mathrm{~km}$ E of Geçitveren, 1270 m, steppe, 16.vi.2014, Armağan 4588 (MA); Ovacık, Güneykonak Köyü, 1230 m, E.Yüce 2805 (R13); Tunceli-Ovacık arası, Munzur Vadisi Milli Parkı çıkışı, 1215 m, 03.vii.2013, E.Yüce 2825 (R13); Munzur Vadisi (R15).

\section{Allium balansae Boiss./Çakılsoğanı}

Mazgirt, Tunceli-Mazgirt arası, Düzgün Baba Dağı, 1940 m, kayalık yamaçlar, 15.vi.2013, E.Vitek, E.Yüce, C.Ergin, H.H.Makal 13-0215 (R13). Ir.-Tur., End.

\section{Allium bingoelense Yıld. \& Ö.Çelik}

Mazgirt, $1 \mathrm{~km} \mathrm{~S}$ of Geçitveren, 1345 m, steppe and oak openings, 16.vi.2014, Armağan 5472 (MA). Ir.Tur., End.

\section{Allium callidictyon C.A.Mey. ex Kunth/Kaya soğanı}

Pertek, 3 km from Elazığ-Pertek-Çemişgezek junction to Çemişgezek (1 km S of Çorovan), 870 m, steppe, 28.vi.2014, Armağan 5124 (MA); Pertek, Pertek's Tunceli exit, $1100 \mathrm{~m}$, rocky areas, 19.vii.2014, Armağan 5264 (MA); above Pertek, 1500 m, D. 31513 (R6); Munzur Vadisi (R15).

\section{Allium cardiostemon Fisch. \& C.A. Mey./ Yamaçkörmeni}

Pülümür, 700 m W of Kırmızı, 1370 m, field border, 05.vi.2014, Armağan 4320 (MA); Ovacık, 12 km from Ovacık to Hozat, $250 \mathrm{~m}$ before from Bilgeç village junction, 1850 m, roadside, 17.vi.2014, Armağan 4738 (MA); Tunceli center, Gözen (iksor) village, 1490 m, field border, 19.vi.2014, Armağan 4982 (MA); Ovacık, S slopes of Kırkmerdivenler, 1200$1700 \mathrm{~m}$, calcereous rocks, stony places and screes, 17.vi.1979, Yıldırımlı 1936 (R4); Pülümür, foots of Silbüs Dağı, 1600-2000 m, clearing of Quercus petraea subsp. pinnatiloba forest, Yıldırımlı 3347 (R4); Ovacık, Ovacık'ın 14 km kuzeydoğusu, 1405 m, taşlık çayırlar, 03.vi.2014, E.Vitek \& E.Yüce 14-
149 (R13); Road Tunceli - Ovacık, c. 5.7 km NW of Tunceli, 940 m, dry meadow, 30.v.2014, V.Y. \& C. 14-048-A (R14); Munzur Vadisi (R15). Ir.-Tur.

\section{Allium cassium Boiss./Keldağaksoğanı}

Pülümür, Karagöl Köyü, Hengirvan Yaylaları, 11.v.2013 E.Yüce 2592 (R13). E.Medit.

\section{Allium chrysantherum Boiss. \& Reut./Sarı kafa}

Pertek to Tunceli, $43 \mathrm{~km}$ from Elazığ, $1400 \mathrm{~m}$, D. 29146 (R6).

\section{Allium colchicifolium Boiss./Gömülgen}

Tunceli center, road of Tunceli - Sütlüce, $14 \mathrm{~km}$ after from Tunceli-Sütlüce junction, 1800 m, steppe scree, 23.v.2014, Armağan 3824 (MA); Tunceli center, $26 \mathrm{~km}$ from Tunceli to Ovacık (Munzur valley), 1100 m, steppe, 30.iv.2015, Armağan 6688 (MA); Tunceli to Ovacık, 1100 m, T. Baytop (R6); Tunceli-Ovacık arası, 1092 m, 05.vii.2012, E.Yüce 2414 (R13); Tunceli-Ovacık arası, Venk Köprüsü çevresi, 1040 m, 05.vii.2012, E.Yüce 2420 (R13). Ir.-Tur

\section{Allium dictyoprasum C.A.Mey. ex Kunth/Top soğan}

Tunceli center, road of Tunceli-Sütlüce, $15 \mathrm{~km}$ after from Tunceli-Sütlüce junction (to $10 \mathrm{~km}$ Çıralı village), $1844 \mathrm{~m}$, roadside, 27.viii.2014, Armağan 6182 (MA). Ir.-Tur.

\section{Allium eginense Freyn/Eğin soğanı}

Tunceli center, $8 \mathrm{~km}$ from Tunceli to Ovacık (Munzur Valley), $945 \mathrm{~m}$, steep steppe slopes, 25.v.2014, Armağan 3959 (MA); Tunceli center, 26 $\mathrm{km}$ from Tunceli to Ovacık (Munzur valley), $1060 \mathrm{~m}$, steppe, 26.v.2014, Armağan 4030 \& 4032 (MA); Hozat, between Karacaköy and Uzundal, road of Sarısaltuk Türbesi, 1730 m, steppe, 17.vi.2014, Armağan 4640 (MA); Hozat, between Karacaköy and Uzundal, around of Sarısaltuk Türbesi, 2270 m, steppe, 17.vi.2014, Armağan 4685 (MA). Ir.Tur., End.

Allium flavum L. subsp. tauricum (Besser ex Rchb.) K.Richt. var. tauricum/Torossarısı

Pertek, 12 km from Pertek to Çemişgezek, around 
of Singeç bridge, $865 \mathrm{~m}$, steppe, 24.v.2014, Armağan 3903 (MA); Ovacık, $1.5 \mathrm{~km}$ from mainroad of Ovacık-Tunceli to Yakatarla village, 1320 m, steppe, 04.vi.2014, Armağan 4214 (MA); Ovacık, Akyayık Köyü, 1315 m, 26.v.2013, E.Yüce 2661 (R13). Medit.

\section{Allium glumaceum Boiss. \& Hausskn./Yırtık soğan}

Ovacık, $3 \mathrm{~km} \mathrm{~N}$ of Işıkvuran (Munzur Mountains), 2146 m, scree slopes, 08.viii.2014, Armağan 5926 (MA). Ir.-Tur., End.

\section{Allium guttatum Stev./Benli soğan}

Nazımiye, between Ramazanköy and Ayranlı, 1420 m, eroded slopes, 23.vii.2014, Armağan 5594 (MA); N. of Pülümür, 2000 m, Pasche 78/19 (R6).

\section{Allium kharputense Freyn \& Sint./Harputsoğanı}

Tunceli center, road of Tunceli - Sütlüce, after $2 \mathrm{~km}$ from junction, $1050 \mathrm{~m}$, steppe, 23.v.2014, Armağan 3809 (MA); Pülümür, 2000 m, It. Leyd. 1959:1616 (R6); Pülümür, foots of Kuzdere Tepesi, steppe, 1500-1700 m, 14.vi.1980, Yıldırımlı 3153 (R4); Altınyüzük Köyü, tarla içi, 08.v.2013, E.Yüce 2553 (R13); Munzur Vadisi (R15). Ir.-Tur.

\section{Allium kunthianum Vved./Kuş körmeni}

Pülümür, between Yalmanlar and Kocatepe, NW of Sarıgül village, 2230 m, steppe, 06.viii.2014, Armağan 5786 (MA); Pülümür, Eğimli - Kocatepe köyü arası, 2341 m, steppe, 06.viii.2014, Armağan 5792 (MA). Ir.-Tur.

\section{Allium myrianthum Boiss./Paksoğan}

Mazgirt, N of Akdüven village, $1110 \mathrm{~m}$, steppe, 16.vi.2014, Armağan 4592 (MA); above Pertek, 1600 m, D. 31056 (R6); Ovacık, Kırkgözeler, towards Ziyaret Tepesi, Munzur Dağları, 16501850 m, 27.vii.1979, Yıldırımlı 2209 (R4); TunceliOvacık arası, Venk Köprüsü çevresi, 1040 m, 05.vii.2012, E.Yüce 2427 (R13). Ir.-Tur.

\section{Allium pallens L. subsp. pallens/Nursoğanı}

Çemişgezek, $2 \mathrm{~km}$ from Vişneli to Arpaderen, $1080 \mathrm{~m}$, field border, roadside, 21.vi.2014,
Armağan 5097 (MA); Elazığ to Erzincan, 1560 m, Buttler 15865 (R6); Tunceli-Ovacık arası, 1092 m, 10.vi.2013, E.Yüce 2785a (R13); Munzur Vadisi (R15). Medit.

\section{Allium paniculatum L. subsp. paniculatum/ Sürüsalkım}

Ovacık, Kırkgözeler, towards Ziyaret Tepesi, Munzur Dağları, 1650-1850 m, 27.vii.1979, Yıldırımlı 2208 (R4); Ovacık, Akyayık Köyü, 1315 m, 10.vi.2013, E.Yüce 2785 (R13); Munzur Vadisi (R15). Medit.

\section{Allium pseudoflavum Vved./Küllüsoğan}

Tunceli center, $10 \mathrm{~km}$ from Tunceli to Ovacık (Munzur Valley), 960 m, steppe, 25.v.2014, Armağan 3987 (MA); Mazgirt, main road of Elazığ - Tunceli, crossroad of Kızılcık, 1060 m, steppe, 02.vi.2014, Armağan 4134 (MA); Nazımiye, 3 km from Nazımiye to Tunceli, $1510 \mathrm{~m}$, oak openings, 03.vi.2014, Armağan 4183 (MA); Pertek, 1500 m, D. 31514 (R6); Tunceli-Ovacık arası, 1092 m, 26.v.2013, E.Yüce 2661a (R13). Ir.-Tur.

\section{Allium purpureoviride Koyuncu \& i.Genç/Renkli soğan}

Pertek, 27 miles from Elazığ to Tunceli, step calcareous south slope, $1400 \mathrm{~m}$ a.s.l., 06.vi.1957, Davis 29146 and Hedge (K) (R12); Pertek, 7 km from Pertek to Tunceli, dry rocky slopes, $1300 \mathrm{~m}$ a.s.I., 08.vi.1990, N. and E. Özhatay (ISTE 61640) (R12). Ir.-Tur., End.

\section{Allium pustulosum Boiss. \& Hausskn./Beysoğanı}

Tunceli center, $10 \mathrm{~km}$ from Aktuluk to Demirkapı, 1450 m, steppe, 21.vii.2014, Armağan 5395 (MA); Ovacık, Mercan Vadisi, 29.v.2012, E.Yüce 2194 (R13); Munzur Vadisi (R15). Ir.-Tur.

\section{Allium roseum L./Gül soğanı}

50 km N.E. of Tunceli, 2000 m, It. Leyd. 1959:1606 (R6).

\section{Allium schoenoprasum L./Peynir sirmosu}

Munzur Da. above Ovacık, 2650 m, D. 31427 (R6); Mazgirt, c. $30 \mathrm{~km}$ ESE of Tunceli, c. $1 \mathrm{~km}$ 
$E$ of Akdüven, $1430 \mathrm{~m}$, dry meadow with rocks, 13.vi.2013, V.Y.E. \& M. 13-0145 (R14); Pülümür, $35.6 \mathrm{~km}$ NNE Tunceli, main road Tunceli - Erzincan, $3.5 \mathrm{~km}$ SW Mezra, $1200 \mathrm{~m}$, roadside and dry slope, 17.vi.2013, V.Y.E. \& M. 13-0332 (R14).

\section{Allium scorodoprasum L. subsp. rotundum (L.) Stearn/Delipırasa}

Pertek, main road Pertek - Çemişgezek, 2.5 km before Singeç bridge, 865 m, steppe, 24.v.2014, Armağan 3889 (MA); Tunceli center, 17 km from Tunceli to Ovacık (Munzur Valley), $1015 \mathrm{~m}$, eroded slopes, 25.v.2014, Armağan 4014 (MA); Tunceli center, Pülümür Valley, $650 \mathrm{~m}$ NW of Kutudere, $1070 \mathrm{~m}$, steppe and oak openings, 03.vi.2014, Armağan 4186 (MA); Tunceli-Ovacık arası, Anafatma civarı, 940 m, 21.v.2012, E.Yüce 2090 (R13); Road Tunceli - Ovacık, c. 5.7 km NW of Tunceli, 940 m, dry meadow, 30.v.2014, V.Y. \& C. 14-048 (R14); Munzur Vadisi (R15).

\section{Allium sintenisii Freyn./Dikenli körmen}

Pülümür, 11 km NW of Ardıçlı (Gersunut), Munzur Mountains, 2516 m, rocky areas, 27.vi.2015, Armağan 6655 (MA). Ir.-Tur., End.

\section{Allium stamineum Boiss./Yabansarımsağı}

Ovacık, $47 \mathrm{~km}$ from Tunceli to Ovacık (Munzur Valley), 1200 m, steppe, 26.v.2014, Armağan 4055 (MA); Ovacık, $51 \mathrm{~km}$ from Tunceli to Ovacık (Munzur Valley), 1210 m, steppe, 26.v.2014, Armağan 4066 (MA); Ovacık, $8 \mathrm{~km}$ from main road of Ovacık Tunceli to Yakatarla, 1680, steppe, 04.vi.2014, Armağan 4227 (MA); Hozat, between Karacaköy and Uzundal, road of Sarısaltuk Türbesi, $1730 \mathrm{~m}$, steppe, 17.vi.2014, Armağan 4639 (MA); TunceliOvacık arası, 1173 m, 27.v.2013, E.Yüce 2674 (R13). E.Medit.

\section{Allium tauricola Boiss./Toros soğanı}

Ovacık, $3 \mathrm{~km} \mathrm{~N}$ of Işıkvuran, Munzur Mountains, 2184 m, scree, 08.viii.2014, Armağan 5927 (MA). Ir.-Tur., End.

\section{Allium tchihatschewii Boiss./Saklı soğan}

Tunceli center, road of Tunceli - Sütlüce, after $500 \mathrm{~m}$ from the junction, $940 \mathrm{~m}$, steppe slopes, 23.v.2014, Armağan 3777 (MA). Ir.-Tur., End.

Allium trachycoleum Wendelbo/Boz sarımsak

Ovacık, $1.5 \mathrm{~km}$ from main road of Ovacık - Tunceli to Yakatarla, 1320 m, steppe, 04.vi.2014, Armağan 4208 (MA). Ir.-Tur.

\section{Allium tripedale Trautv./Şahsarımsağı}

Ovacık, Munzur Gözeleri çevresi, 08.v.2012, E.Yüce 2998 (R13).

\section{Allium tuncelianum (Kollmann) Özhatay,} B.Mathew \& Siraneci/Tuncelisarımsağı

Ovacık, W of Ziyaret (Munzur Gözeleri), 1340 m, rocky areas, 20.vi.2014, Armağan 4983 (MA); Munzur Da., in Aksu Dere above Ovacık, 1800 m, 21.vii.1957, Davis 31498 (R6); Tunceli to Ovacık, 1100 m, T. Baytop ISTE 23198 (R6); Munzur Dag, Aksu Dere above Ovacık, 1800 m, 21.vii.1957, Davis 31498 (R8); Tunceli-Ovacık arası, Anafatma civarı, 910 m, 05.vii.2012, E.Yüce 2401 (R13); Tunceli-Ovacık arası, Anafatma'ya varmadan, 914 m, 05.vii.2012, E.Yüce 2411 (R13); Tunceli-Ovacık arası, 1173 m, 05.vii.2012, E.Yüce 2431 (R13); Munzur Vadisi (R15). Ir.-Tur., End.

\section{Allium vineale L./Sirmo}

Ovacık, 1200 m, 25.vii.1979, Yıldırımlı 2077 (R4); Tunceli-Ovacık arası, Ovacık'a 20 km kala, roadside, 03.vii.2013, E.Yüce 2820 (R13); Munzur Vadisi (R15).

Sternbergia clusiana (Ker Gawl.) Ker Gawl. ex Spreng./Vargetgülü

Ovacık, W of Ovacık, side of Munzur river, 1240 m, meadow, 21.ix.2014, Armağan 6342 (MA); Ovacık, 1200 m, 27.x.1980, Yıldırımlı 4106 (R4); TunceliOvacık arası, Geyiksuyu Köyü yol ayırımından 2-3 km, roadside, 27.v.2013, E.Yüce \& i..Eker 2701 (R13); Munzur Vadisi (R15). Ir.-Tur.

\section{ARACEAE}

\section{Arum orientale M.Bieb./Yaldıran}

Pülümür, between köprü and Yeşilköy, clearing of Quercus petraea subsp. pinnatiloba forest, 15001600 m, 15.vi.1980, Yıldırımlı 3289 (R4); Tunceli- 
Ovacık arası, Halbori Gözeleri, 08.v.2013, E.Yüce 2561 (R13); Munzur Vadisi (R15). Euro.-Sib. (Eux.).

Arum rupicola Boiss. var. rupicola (= Arum conophalloides Kotschy ex Schott)/Dağsorsalı

Ovacık, 1.5 km E of Işıkvuran, 1960 m, steppe, 04.vi.2014, Armağan 4315 (MA); Pülümür, Kocatepe Köyü, 1625 m, 02.vi.2014, E.Vitek, E.Yüce, U.Çakılcıoğlu 14-128 (R13). Ir.-Tur., End.

Arum rupicola Boiss. var. virescens (Stapf) P.C.Boyce/Dağsorsalı

Ovacık, $44 \mathrm{~km}$ from Tunceli to Ovacık (Munzur Valley), 1190 m, humid places, 26.v.2014, Armağan 4043 (MA); Ovacık, S slopes of Kırkmerdivenler, calcereous rocks, stony places and screes, 12001700 m, 17.vi.1979, Yıldırımlı 1928 (R4). Ir.-Tur.

\section{Biarum carduchorum (Schott) Engl./Kardi}

Tunceli, Sakaltutan Da., 10 km N.E. of Pertek, Watson et al. 2404 (R6); Ovacık, above Paşadüzü Köyü, Korti Deresi, Tepesi and Yaylası, 12502100 m, 28.x.1980, Yıldırımlı 4107 (R4); Ovacık, Güneykonak Köyü, 1230 m, 27.ix.2013, E.Yüce 2879 (R13); Munzur Vadisi (R15).

Eminium rauwolffii (Blume) Schott var. rauwolffii /Yılanbacağı

Tunceli-Ovacık arası, Anafatma civarı, 940 m, 03.v.2013, E.Yüce 2531 (R13). Ir.-Tur.

\section{ASPARAGACEAE}

Bellevalia anatolica B.Mathew \& Özhatay/Yamaç Sümbülü

N. of Elazığ near Pertek, on road to Tunceli, rocky hillside, 04.vi.1987, fl. cult. Kew, 10.iv.1990, B. Mathew 11033 (R8). Ir.-Tur., End.

\section{Bellevalia fominii Woronow/Benlisümbül}

Ovacık, $3 \mathrm{~km} \mathrm{~N}$ of Işıkvuran, Munzur Mountains, 2080 m, alpine steppe, 04.vi.2014, Armağan 4256 (MA); Ovacık, Akyayık Köyü, 1315 m, 21.v.2013, E.Yüce 2737 (R13). Ir.-Tur.

Bellevalia gracilis Feinbrun/Aktepeli
Tunceli center, $15 \mathrm{~km}$ from Tunceli to Ovacık (Munzur Valley), steppe slopes, 11.iv.2015, Armağan 6408 (MA); Ovacık, nr Munzur river, 1200 m, 05.v.1979, Yıldırımlı 1313 (R6); TunceliOvacık arası, 45. km, 08.v.2012, E.Yüce 2042a (R13); Tunceli-Ovacık arası, Munzur Vadisi Milli Parkı çıkışı, 1215 m, 20.iv.2012, E.Yüce 2053 (R13); Munzur Vadisi (R15). Ir.-Tur., End.

\section{Bellevalia kurdistanica Feinbrun/Alacasümbül}

Tunceli-Ovacık arası, Siliç Köprüsü çevresi, 09.iv.2013, E.Yüce 2484 (R13). Ir.-Tur.

\section{Bellevalia longipes Post/Saçaksümbül}

Mazgirt, on road Elazığ - Tunceli, $2 \mathrm{~km}$ after from Beylermezrası, 855 m, steppe scree, 02.vi.2014, Armağan 4155 (MA); Tunceli center, on road Tunceli - Sütlüce, $2 \mathrm{~km}$ after crossroad, $1050 \mathrm{~m}$, steppe, 23.v.2014, Armağan 3810 (MA); TunceliOvacık arası, 45. km, 08.v.2012, E.Yüce 2042 (R13); Atlantı (Pah köprüsü), 935 m, 31.v.2012, E.Yüce 2285 (R13); Tunceli-Ovacık arası, Anafatma civarı, 940 m, 22.iv.2013, E.Yüce 2532 (R13); TunceliOvacık arası, 40. km, 03.v.2013, E.Yüce 2537 (R13); Tunceli-Ovacık arası, Dedeağaç Köyü, 1370 m, 14.v.2013, E.Yüce 2606 (R13). Ir.-Tur.

Bellevalia paradoxa (Fisch. \& C.A.Mey.) Boiss. (= Bellevalia pycnantha (K.Koch) Losinsk.)/ Aşpenceri

Ovacık, around Beşevler, at the junction of Munzur Suyu and Mercan Çayı, 1100 m, 07.v.1979, Yıldırımlı 1396 (R4); Tunceli-Ovacık arası, Munzur Vadisi Milli Parkı çıkışı, 1215 m, 03.v.2013, E.Yüce 2518 (R13); Munzur Vadisi (R15). Ir.-Tur.

Bellevalia speciosa Woronow ex Grossh. (= Bellevalia sarmatica (Pall. ex Miscz.) Woronow)/ Saplısümbül

Tunceli to Pülümür, $9.6 \mathrm{~km}$ from Pülümür, D. 29206 (R6); Tunceli-Ovacık arası, Anafatma civarı, 910 m, 08.v.2012, E.Yüce 2061 (R13).

Hyacinthella acutiloba K. Perss. \& Wendelbo/ Sivri sümbül

Ovacık, Karagöl vadisi, Munzur dağları, 1400 m, 
05.v.1979, Yıldırımlı 1327 (R4). Ir.-Tur., End.

Hyacinthus orientalis L. subsp. chionophilus Wendelbo/Kopça

Ovacık, $3 \mathrm{~km} \mathrm{~N}$ of Işıkvuran, Munzur Mountains, 2020 m, rocky areas, 04.vi.2014, Armağan 4254 (MA); Tunceli center, $14 \mathrm{~km}$ from Tunceli to Ovacık (Munzur Valley), 1000 m, steppe slopes, 11.iv.2015, Armağan 6384 (MA); Munzur Da. above Ovacık, 2500 m, T. Baytop ISTE 24243 (R6); Ovacık, Karagöl vadisi, Munzur dağları, 1400 m, 05.v.1979, Yıldırımlı 1327 (R4); Tunceli-Ovacık arası, Munzur Vadisi Milli Parkı çıkışı, 1215 m, 20.iv.2012, E.Yüce 2035 (R13); Tunceli-Ovacık arası, Venk Köprüsü'ne varmadan roadside, 20.iv.2012, E.Yüce 2027 (R13); Ovacık, Akyayık Köyü, 1315 m, 31.iii.2013, E.Yüce 2478 (R13); Tunceli-Ovacık arası, Munzur Vadisi Milli Parkı çıkışı, 1215 m, 09.iv.2013, E.Yüce 2486 (R13); Ovacık, Güneykonak Köyü, 1230 m, 08.iv.2013, E.Yüce 2500 (R13); Munzur Vadisi (R15). Ir.-Tur., End.

\section{Muscari armeniacum Leichtlin ex Baker/ Gâvurbașı}

Pülümür, 12 km NW of Ardıçlı (Gersunut), on Munzur Mountains, 2490 m, steppe scree, 27.vi.2015, Armağan 6670 (MA); Tunceli center, Örenönü Nature Park (Pülümür Valley), 920 m, meadows, 12.iv.2015, Armağan 6410 (MA); Pülümür, $9 \mathrm{~km}$ from Pülümür to Tunceli (Pülümür Vallay), S of Kangallı, 1324 m, steppe, 05.vi.2014, Armağan 4429 (MA); Ovacık, 3km N of Işıkvuran, Munzur Mountains, $2100 \mathrm{~m}$, alpine steppe, 04.vi.2014, Armağan 4269 (MA); Ovacık, banks of Munzur Suyu, 1200 m, 05.v.1979, Yıldırımlı 1321 (R4); Tunceli-Ovacık arası, Anafatma civarı, 940 m, 08.v.2012, E.Yüce 2060 (R13); Tunceli-Ovacık arası, Venk Köprüsü çevresi, 1040 m, 08.v.2012, E.Yüce 2081 (R13); Tunceli-Ovacık arası, Anafatma ziyareti üst tarafı, 03.v.2013, E.Yüce 2523 (R13); Tunceli-Ovacık arası, Anafatma Ziyareti çevresi, 916 m, 03.v.2013, E.Yüce 2528 (R13); Munzur Vadisi (R15).

\section{Muscari aucheri (Boiss.) Baker/Gök müșkürüm}

Ovacık, banks of Munzur Suyu, 1200 m, 05.v.1979, Yıldırımlı 1320 (R4); Munzur Vadisi (R15). End.

\section{Muscari comosum (L.) Mill./Morbaş}

Tunceli center, on road of Tunceli - Pertek, $1 \mathrm{~km}$ after from Kopuzlar, 1114 m, steppe, 22.v.2014, Armağan 3755 (MA); Pülümür, foots of Kuzdere Tepesi, steppe, 1500-1700 m, 14.vi.1980, Yıldırımlı 3153 (R4); Tunceli-Ovacık arası, Avsugar Köprüsü çevresi, 995 m, 29.v.2012, E.Yüce 2148 (R13); Tunceli-Ovacık arası, Munzur Vadisi Milli Park çıkışı, 1215 m, 29.v.2012, E.Yüce 2168 (R13); TunceliOvacık arası, Dedeağaç Köyü, 1370 m, 14.v.2013, E.Yüce 2605 (R13); Ovacık, Güneykonak Köyü, 1230 m, 10.v.2013, E.Yüce 2616 (R13); TunceliOvacık arası, Halbori Gözeleri, 26.v.2013, E.Yüce 2664 (R13); Mazgirt, c. 30 km ESE of Tunceli, c. 1 $\mathrm{km} \mathrm{E}$ of Akdüven, $1430 \mathrm{~m}$, dry meadow with rocks, 01.vi.2013, V.Y.E. \& M. 13-0147 (R14); Pülümür, c. $38 \mathrm{~km}$ NNE Tunceli, road to Kocatepe, $0.9 \mathrm{~km} \mathrm{SE}$ Kovuklu, 1625 m, Astragalus spiny shrub steppe, 02.vi.2014, V.Y. \& U. 14-133b (R14); Nazımiye, c. 27 km NE of Tunceli, above village Büyükyurt, 1775 m, dry slope, 16.vi.2013, V.Y. \& E. 13-0238 (R14). Medit.

\section{Muscari longipes Boiss./Buğulu sümbül}

Pertek, between Pertek and Mercimek, 1280 m, eroded slopes, 26.v.2017, Armağan 7458 (MA); Pertek to Tunceli, $42 \mathrm{~km}$ from Elazığ, $1400 \mathrm{~m}$, D. 29112 (R6).

\section{Muscari massayanum C.Grunert/Şah müşkürüm}

N. of Pülümür, 2000 m, Pasche 78/18 (cult.E) (R6).

\section{Muscari neglectum Guss. ex Ten./Arapüzümü}

Pülümür, $500 \mathrm{~m} \mathrm{~N}$ of Balpayam (3-4 km S of Kırklar), 2400 m, 18.vi.2014, Armağan 4843 (MA); Merkez, Atatürk Mahallesi, 920 m, 20.iv.2012, E.Yüce 2015 (R13); Ovacık, Güneykonak Köyü, 1230 m, 21.iv.2013, E.Yüce 2639 (R13).

\section{Ornithogalum alpigenum Stapf/Akyıldız}

Nazımiye, Dereova township, $5 \mathrm{~km}$ SE of Aşağımarkosör, $2144 \mathrm{~m}$, near of melted snow, pastures, 01.vi.2015, Armağan 6525 (MA); Tunceli-Ovacık arası, Venk Köprüsü çevresi, 1040 m, 21.iv.2012, E.Yüce 2093 (R13); TunceliOvacık arası, 40. km, 21.iv.2012, E.Yüce 2095 (R13); Tunceli-Ovacık arası, Munzur Vadisi Milli Parkı çıkışı, 1215 m, 29.v.2012, E.Yüce 2167 (R13); Tunceli-Ovacık arası, Anafatma civarı, 940 m, 
05.vii.2012, E.Yüce 2441 (R13). E.Medit., End.

\section{Ornithogalum arcuatum Steven/Kurtkiriși}

Ovacık, $10 \mathrm{~km}$ from Ovacık to Hozat, junction of Kuşluca village, 1710 m, pastures, 17.vi.2014, Armağan 4745 (MA); Hozat, 700 m N of Uzundal, 1700 m, steppe, 17.vi.2014, Armağan 4690 (MA); Mazgirt, main road of Elazığ - Tunceli, $2 \mathrm{~km}$ after from Beylermezrası, $855 \mathrm{~m}$, steppe, eroded hill, 02.vi.2014, Armağan 4154 (MA); Munzur Da. above Ovacık, 2400 m, D. 31377 (R6); Ovacık, around Munzur Suyu, 1200 m, 06.vii.1980, Yıldırımlı 3439 (R4); Tunceli-Ovacık arası, Işıkvuran Köyü çıkışı, 26.v.2013, E.Yüce \& I.Eker 2675 (R13); Munzur Vadisi (R15). Ir.-Tur.

\section{Ornithogalum cuspidatum Bertol./Bitik sasal}

Tunceli center, $16 \mathrm{~km}$ from Tunceli to Ovacık (Munzur Valley), $980 \mathrm{~m}$, pastures in stream side, 25.v.2014, Armağan 4008 (MA); Ovacık, 48 km from Tunceli to Ovacık (Munzur Valley), 1190 $m$, pastures in stream side, 26.v.2014, Armağan 4056 (MA).

\section{Ornithogalum hajastanum Agapova/Çarpık sasal}

Ovacık, $3 \mathrm{~km} \mathrm{~N}$ of Işıkvuran, Munzur Mountains, 1980 m, steppe, 19.vi.2014, Armağan 4946 (MA); Ovacık, $8 \mathrm{~km}$ from Eskigedik to Işıkvuran, $2120 \mathrm{~m}$, steppe, 19.vi.2014, Armağan 4926 (MA); Ovacık, $17 \mathrm{~km}$ from Ovacık to Hozat, E of Halitpınar, 1895 m, steppe, 17.vi.2014, Armağan 4718 (MA); Hozat, between Karacaköy and Uzundal, around of Sarısaltuk Türbesi, 2270 m, rocky areas, 17.vi.2014, Armağan 4684 (MA); Ovacık, 5 km main road of Ovacık - Tunceli to Yakatarla, $1620 \mathrm{~m}$, steppe, 04.vi.2014, Armağan 4217 (MA); Tunceli center, $26 \mathrm{~km}$ from Tunceli to Ovacık (Munzur Valley), 1060 m, steppe, 26.v.2014, Armağan 4031 (MA); Ovacık, $44 \mathrm{~km}$ from Tunceli to Ovacık arası (Munzur Valley), $1190 \mathrm{~m}$, in the gardens, 26.v.2014, Armağan 4047 (MA).

\section{Ornithogalum Iuschanii Stapf./Çayır sasalı}

Tuncelicenter, $18 \mathrm{~km}$ from Tuncelito Ovacık (Munzur Valley), 915 m, steppe, 17.iv.2015, Armağan 6698 (MA); Pülümür, 12 km NW of Ardıçlı (Gersunut), Munzur Mountains, 2490 m, near to melted snow, 27.vi.2015, Armağan 6672 (MA); Pülümür, 11 km NW of Ardıçlı (Gersunut), Munzur Mountains, 2510 m, rocky steppe, 18.vi.2014, Armağan 4888 (MA). End.

\section{Ornithogalum macrum Speta/Yer sasalı}

Tunceli center, $700 \mathrm{~m}$ from Demirkapı to Çılga, $1410 \mathrm{~m}$, in the gardens, 06.vi.2014, Armağan 4437 (MA). E.Medit., End.

\section{Ornithogalum montanum Cirillo/Dağ yıldızı}

Ovacık, 27.5 km NNW Tunceli, road AşağıtorunobaAgdat, 1825 m, semihumid meadow, 18.vi.2013, V.Y.E. \& M. 13-0374 (R14); Ovacık, 27.5 km NNW Tunceli, road Aşağıtorunoba - Agdat, 1825 m, semihumid meadow, 18.vi.2013, V.Y.E. \& M. 130375a (R14). E.Medit.

\section{Ornithogalum munzurense Speta/Munzur yıldızı}

Tunceli center, on road Tunceli - Çıralı, $17 \mathrm{~km}$ from crossroad of Tunceli - Sütlüce (to $8 \mathrm{~km}$ Çıralı), 1810 m, steppe, 01.vi.2015, Armağan 6520 (MA); 19 km from Tunceli along Munzur-River towards Ovacık, steep scree-slopes and cliff-ledges, calcareous rock, 950 m, 11.iv.1990, E.Pasche KPPZ 90205, cult. LI: 21.v.1993, 18.v.1992 (R11). End.

\section{Ornithogalum narbonense L./Akbaldır}

Ovacık, $1.5 \mathrm{~km}$ from main road of Ovacık-Tunceli to Yakatarla, 1320 m, steppe, 04.vi.2014, Armağan 4215 (MA); Tunceli-Ovacık arası, Işıkvuran Köyü yol ayrımından 6-7. km, 26.v.2013, E.Yüce \& i.Eker 2673 (R13); Ovacık, Munzur Vadisi Milli Parkı çıkışı, 1215 m, 27.v.2013, E.Yüce \& i..Eker 2705 (R13). Medit.

\section{Ornithogalum oligophyllum E.D.Clarke/ Kurtsoğanı}

Ovacık, banks of Munzur Suyu, 1200 m, 05.v.1979, Yıldırımlı 1319 (R4); Ovacık (Merkez), Yeşilyazı, 1275 m, 08.v.2012, E.Yüce 2072 (R13); TunceliOvacık arası, Halbori gözeleri, 08.v.2013, E.Yüce 2554 (R13); Munzur Vadisi (R15).

Ornithogalum orthophyllum Ten. subsp. kochii (Parl.) Zahar/Bayıryııdızı

Tunceli-Ovacık arası, Halbori gözeleri, 08.v.2013, 
E.Yüce 2554 (R13); Ovacık, Akyayık Köyü, 1315 m, 17.v.2013, E.Yüce 2599 (R13); Tunceli-Ovacık arası, Halbori gözeleri, 26.v.2013, E.Yüce \& I Eker 2662 (R13); Tunceli-Ovacık arası, Geyiksuyu Köyü yol ayrımından 2. km, dağ yamacı, 27.v.2013, E.Yüce \& i Eker 2700 (R13).

Ornithogalum persicum Hausskn. ex Bornm./ Karayıldız

Pertek, $4 \mathrm{~km}$ from Pertek to Tunceli, 1260 m, steppe, 03.vi.2014, Armağan 7523 (MA); Pülümür, 2 km from Turnadere to Közlüce, 1560 m, steppe, 18.vi.2014, Armağan 4773 (MA); Hozat, between Çı̆̆ırlı and Dervişcemal, the crossroad of Buzlupınar, 1740 m, steppe, 17.vi.2014, Armağan 4634 (MA); Ovacık, 8 km from Aşağıtorunoba to Ağaçpınar, 1800 m, steppe, 04.vi.2014, Armağan 4308 (MA); Tunceli center, between Tunceli and Pülümür (Pülümür Valley), 650 m NW of Kutudere, 1070 m, steppe, 03.vi.2014, Armağan 4185 (MA); Pertek to Tunceli, 1350 m, D. 29156 (R6); OvacıkKöseler Köyü arası, 17.vi.2013, E.Yüce 2882 (R13); Munzur Vadisi (R15). Ir.-Tur.

\section{Ornithogalum pyrenaicum L./Eșeksusamı}

Pertek to Tunceli, 40 km from Elazı̆̆, 1400 m, D. 29106 (R6); Tunceli-Ovacık arası, Işıkvuran Köyü, 19.v.2013, E.Yüce 2763 (R13); Ovacık-Munzur gözeleri arası, Ada Köyü civarı, 1285 m, 03.vii.2013, E.Yüce 2829 (R13); Ovacık, c. 18.3 km NE of Ovacık, c. $0.5 \mathrm{~km}$ NNE of small river dam, near locality called Şahverdi, $1500 \mathrm{~m}$, stony meadow, 03.vi.2014, V.Y. 14-155 (R14); Ovacık, road TunceliOvacık, c. $6.2 \mathrm{~km}$ EESE Ovacık, $0.2 \mathrm{~km}$ W of bridge at mouth of side river, $1215 \mathrm{~m}$, meadow, 03.vi.2014, V.Y. 14-146 (R14).

\section{Ornithogalum sphaerocarpum A.Kern./ Salkımsakarca}

Ovacık, $47 \mathrm{~km}$ from Tunceli to Ovacık (Munzur Valley), 1200 m, steppe, 26.v.2014, Armağan 4054 (MA); Tunceli-Ovacık arası, Anafatma civarı, 940 m, 29.v.2012, E.Yüce 2212 (R13); Ovacık, Akyayık Köyü, 1315 m, 25.v.2013, E.Yüce 2731 (R13); TunceliOvacık arası, Munzur Vadisi Milli Parkı çıkışı, 1215 m, 03.vii.2013, E.Yüce 2827 (R13); Pülümür, 39 km NE Tunceli, road to Kocatepe, southern part of Kovuklu, 1550 m, meadow, 17.vi.2013, V.Y.E. \&
M. 13-0297 (R14); Ovacık, 27.5 km NNW Tunceli, road Aşağıtorunoba - Agdat, 1825 m, semihumid meadow, 18.vi.2013, V.Y.E. \& M. 13-0379 (R14); Ovacık, road Tunceli - Ovacık, c. 6.6 km EESE Ovacık, $0.35 \mathrm{~km}$ SE of bridge at mouth of side river, $1230 \mathrm{~m}$, humid meadow, 12.vi.2013, V.Y. E. \& M. 13-0023 (R14).

\section{Ornithogalum umbellatum L. / Sunbula}

Tunceli-Ovacık arası, Munzur Vadisi Milli Parkı çıkışı, 1215 m, 08.v.2012, E.Yüce 2085 (R13); Tunceli-Ovacık arası, Anafatma civarı, 940 m, 03.v.2013, E.Yüce 2529 (R13); Tunceli-Ovacık arası, Anafatma ziyareti, Munzur çayı kenarı, yolun alt kısmı, 03.v.2013, E.Yüce 2550 (R13).

\section{Puschkinia scilloides Adams/Serhişing}

Pülümür, 20 km from Pülümür to Tunceli (Pülümür Valley), $1215 \mathrm{~m}$, pastures near to melted snow, 12.iv.2015, Armağan 6392 (MA); Ovacık, around Beşevler, at the junction of Munzur Suyu and Mercan Çayı, 1100 m, 07.v.1979, Yıldırımlı 1397 (R4); Ovacık, Akyayık Köyü, 1315 m, 22.iv.2013, E.Yüce 2574 (R13); Ovacık, Güneykonak Köyü, 1230 m, 01.v.2013, E.Yüce 2621 (R13); Ovacık, Güneykonak Köyü, 1230 m, 14.iv.2013, E.Yüce 2651 (R13); Munzur Vadisi (R15). Ir.-Tur.

\section{Scilla bifolia L./Ormansümbülü}

Ovacık, $8 \mathrm{~km}$ from Ovacık to Tunceli, $500 \mathrm{~m} \mathrm{~N}$ of Yaylagünü, $1212 \mathrm{~m}$, pastures in stream side, 11.iv.2015, Armağan 6406 (MA); Tunceli-Ovacık arası, Munzur Vadisi Milli Parkı çıkışı, 1215 m, 20.iv.2012, E.Yüce 2033 (R13); Ovacık, Akyayık Köyü, 1315 m, 22.iv.2013, E.Yüce 2569-2574 (R13); Ovacık, Güneykonak Köyü, 1230 m, 11.iv.2013, E.Yüce 2649 (R13); Tunceli-Ovacık arası, Munzur Vadisi Milli Parkı çıkışı, 1215 m, 27.v.2013, E.Yüce \& i.Eker 2706 (R13). Medit.

Scilla siberica Haw. subsp. armena (Gross.) Mordak/Camışkıran

Pülümür, $46 \mathrm{~km}$ from Tunceli to Pülümür (Pülümür Valley), $1215 \mathrm{~m}$, pastures near to melted snow, 12.iv.2015, Armağan 6389 (MA); Ovacık, 3 km N of Işıkvuran, Munzur Mountains, 2020 m, rocky crevices, 04.vi.2014, Armağan 4244 (MA); Ovacık, Munzur Dağları, Mercan Boğazı, in front of 
Şahverdi Köyü, 1500-1800 m, 08.v.1979, Yıldırımlı 1438 (R4); Tunceli-Ovacık arası, Dilek Köyü girişi, roadside, 1110 m, 12.iv.2012, E.Yüce 2010 (R13); Tunceli-Ovacık arası, Anafatma ziyaretinin üst tarafı, 12.iv.2012, E.Yüce 2011 (R13); Tunceli-Ovacık arası, Geyiksuyu karakolu çevresi, 20.iv.2012, E.Yüce 2016 (R13); Tunceli-Ovacık arası, Venk Köprüsüne varmadan, 20.iv.2012, E.Yüce 2029 (R13); Ovacık, Akyayık Köyü, 1315m, 22.iv.2013, E.Yüce 2564 (R13). Ir.-Tur.

\section{BUTOMACEAE}

\section{Butomus umbellatus L./Bataklıkgülü}

Mazgirt, Yukarıoyumca village, 1430 m, sulak alanlar, 15.viii.2014, Armağan 6055(MA); Mazgirt, Yukarıoyumca, 1430 m, 11.vi.2013, E.Vitek, E.Yüce, C.Ergin, H.H.Makal 13-0018 (R13). Euro.-Sib.

\section{COLCHICACEAE}

\section{Colchicum kotschyi Boiss./Acı çiğdem}

Tunceli center, $4 \mathrm{~km}$ from Gürbüzler to Günlüce (N of Kıl village), steppe, 01.ix.2014, Armağan 6218 (MA). Ir.-Tur.

\section{Colchicum munzurense K.Perss. / Munzurçiğdemi}

Ovacık, $52 \mathrm{~km}$ from Tunceli to Ovacık (Munzur Valley), N of Yaylagünü, $1212 \mathrm{~m}$, steppe, 11.iv.2015, Armağan 6387 (MA); $19 \mathrm{~km}$ from Tunceli to Ovacık along Munzur river, steep scree slopes and cliff ledges, calcareous rock, 950 m, 11.iv.1990, Kammerl., Pasche, Perss. \& Zetterl. 90-193 (R8); $27 \mathrm{~km}$ from Tunceli to Ovacık along Munzur river, 1000 m, Kammerl. \& al. 90-208 (R8); TunceliOvacık arası, Anafatma ziyareti üst kısmı, Taht Köyü, 26.iii.2012, E.Yüce 2000 (R13); TunceliOvacık arası, Munzur Vadisi Milli Parkı çıkışı, 1215 m, 20.iv.2012, E.Yüce 2037, 2038 (R13). Ir.-Tur., End.

\section{Colchicum speciosum Steven/Şepart}

Munzur Vadisi (R15). Euro.-Sib.

Colchicum szovitsii Fisch \& C.A.Mey. subsp. szovitsii (= Colchicum nivale (Boiss. \& A.Huet) Boiss. \& A.Huet ex Stef.)/Katırçiğdemi

Ovacık, $52 \mathrm{~km}$ from Tunceli to Ovacık (Munzur
Valley), N of Yaylagünü, $1212 \mathrm{~m}$, steppe, 11.iv.2015, Armağan 6386 (MA); Ovacık, around Beşevler, at the junction of Munzur Suyu and Mercan Çayı, 1100 m, 07.v.1979, Yıldırımlı 1398 (R4); Tunceli-Ovacık arası, Venk Köprüsü çevresi, 1040 m, 20.iv.2012, E.Yüce 2024 (R13); Munzur Vadisi (R15). Ir.-Tur.

\section{Colchicum triphyllum Kunze/Öksüzali}

Munzur Da. above Ovacık, Karagöl vadisi, 1400 m, 05.v.1979, Yıldırımlı 1326 (R6); Ovacık, Munzur Dağları, around Kankala, above Karataş Köyü, 1800-2000 m, 08.v.1979, Yıldırımlı 1489 (R4); Munzur Vadisi (R15). Medit.

\section{IRIDACEAE}

Crocus biflorus Mill. subsp. tauri (Maw) B.Mathew/ Berfan

Ovacık, $52 \mathrm{~km}$ from Tunceli to Ovacık (Munzur Valley), N of Yaylagünü, $1212 \mathrm{~m}$, steppe, 11.iv.2015, Armağan 6388 (MA); Ovacık, Karagöl vadisi, Munzur dağları, 1400 m, 05.v.1979, Yıldırımlı 1334 (R4); Tunceli-Ovacık arası, Dilek Köyü girişi, 12.iv.2012, E.Yüce 2009 (R13); TunceliOvacık arası, Munzur Vadisi Milli Parkı çıkışı, 1215 m, 20.iv.2012, E.Yüce 2014, 2039 (R13); Munzur Vadisi (R15). Ir.-Tur.

Crocus kotschyanus K.Koch subsp. cappadocicus B.Mathew/Periçiğdemi

Hozat, between Karacaköy and Uzundal, road of Sarısaltuk Türbesi, 1800 m, steppe, 22.ix.2014, Armağan 6353 (MA); Ovacık, 8 km from Işıkvuran to Ağaçpınar, 2150 m, steppe, 21.ix.2014, Armağan 6340 (MA); Pülümür, 4 km N of Kırklar, 2300 m, steppe, 20.ix.2014, Armağan 6312 (MA); Pülümür, $11 \mathrm{~km}$ NW of Ardıçlı (Gersunut), Munzur Mountains, 2240 m, steppe, 20.ix.2014, Armağan 6287 (MA); Seteri to Pülümür $2000 \mathrm{~m}$, Watson et al. 2401 (R6); Ovacık, above Paşadüzü Köyü, Korti Deresi, Tepesi and Yaylası, 1250-2100 m, 28.x.1980, Yıldırımlı 4108 (R4); Ovacık, Güneykonak Köyü, 1230 m, 16.ix.2013 E.Yüce 2880 (R13). Ir.-Tur., End.

Crocus kotschyanus K.Koch subsp. kotschyanus / Gezgin çiğdem

Munzur Vadisi (R15). 


\section{Gladiolus atroviolaceus Boiss./Kıraçsüseni}

Tunceli center, between Tunceli and Hozat, 3.5 km from Demirkapı to Hozat, 1760 m, 23.v.2014, Armağan 3855 (MA); Ovacık, Karagöl Vadisi, 1300-1550 m, rocky and screes, 07.vii.1980, Yıldırımlı 3515 (R4); Tunceli-Ovacık arası, Yukarı Torunoba civarı, 1190 m, 29.v.2012, E.Yüce 2197 (R13); Tunceli-Ovacık arası, Venk Köprüsü çevresi, 1040 m, 05.viii.2012, E.Yüce 2426 (R13); TunceliOvacık arası, Işıkvuran Köyü, 18.v.2013, E.Yüce 2812 (R13); Tunceli-Ovacık arası, Halbori gözeleri, 11.vii.2013, E.Yüce 2840 (R13); Tunceli center, c. $14.1 \mathrm{~km} \mathrm{NE}$ of Tunceli, road D882 to Gökçek, 2.2 km SSW Gökçek, locality called Kutudere, 1000 $\mathrm{m}$, dry meadows and oak forest, 18.v.2011, V.E. 110096 (R14); Munzur Vadisi (R15). Ir.-Tur.

\section{Gladiolus illyricus W.D.J.Koch/Osman çiçeği}

Nazımiye, $700-900 \mathrm{~m}$ from Aşağırabat village to Büyükyurt (Hakis), 1070 m, steppe, 30.vi.2014, Armağan 5228 (MA); 10 km NW Tunceli, valley of river Munzur, $1.4 \mathrm{~km}$ WSW Dilek, $1005 \mathrm{~m}$, stony slope, 18.vi.2013, V.Y.E. \& M. 13-0409 (R14).

\section{Gladiolus italicus Mill./Kılıçotu}

Tunceli center, road of Tunceli - Elazığ, 700 $\mathrm{m}$ after from Aktuluk, $930 \mathrm{~m}$, oak openings, 22.v.2014, Armağan 3687 (MA); Ovacık, S slopes of Kırkmerdivenler, calcereous rocks, stony places and screes, 1200-1700 m, 17.vi.1979, Yıldırımlı 1929 (R4); Ovacık, Güneykonak Köyü, 1210 m, 10.v.2013, E.Yüce 2615 (R13); Tunceli-Ovacık yolu, Işıkvuran Köyü çıkışı, 26.v.2013, E.Yüce \& i.Eker 2676 (R13); Tunceli-Ovacık yolu, Geyiksuyu Köyü yol ayrımı, dağ yamacı, 27.v.2013, E.Yüce \& i.Eker 2699 (R13); Tunceli center, c. $13.6 \mathrm{~km}$ ESE of Tunceli, road Çukur - Güleç, $3 \mathrm{~km} \mathrm{~S}$ of Çukur, 1435 m, roadside in oak forest, 13.vi.2013, V.Y.E. \& M. 13-0141 (R14); Munzur Vadisi (R15).

\section{Gladiolus kotschyanus Boiss./Çayır kılıçotu}

Tunceli center, around of Kocalar, 1083 m, steppe scree, 12.v.2015, Armağan 6680 (MA); Nazımiye, c. $32 \mathrm{~km}$ NE of Tunceli, NE of Büyükyurt, road to former village Hormek, small side valley above ruines of village, $1820 \mathrm{~m}$, meadow besides rivulet, 01.vi.2014, V.Y. \& U. 14-090 (R14). Ir.-Tur.

\section{Iris aucheri (Baker) Sealy/Kayanavruzu}

Nazımiye, $40 \mathrm{~km}$ from Tunceli to Pülümür (Pülümür Valley), 1150 m, steppe slopes, 12.iv.2015, Armağan 6391 (MA); Ovacık, banks of Munzur Suyu, 1200 m, 05.v.1979, Yıldırımlı 1309 (R4); Tunceli-Ovacık arası, Anafatma civarı, 940 m, 20.iv.2012, E.Yüce 2018 (R13); Ovacık (Merkez), Yeşilyazı, 1275 m, 08.v.2012, E.Yüce 2049, 2050, 2051 (R13); Munzur Vadisi (R15). Ir.-Tur.

Iris caucasica Hoffm. subsp. turcica B. Mathew/ Türknavruzu

Ovacık, Mollaaliler village (Mercan Valley), 1420 m, steppe, 09.v.2015, Armağan 6679 (MA); Pülümür, $3 \mathrm{~km}$ from Sarıgül to Yalmanlar, $2250 \mathrm{~m}$, steppe, 05.vi.2014, Armağan 4367 (MA); Ovacık, Akyayık Köyü, 1315 m, 31.iii.2013, E.Yüce 2572 (R13); Munzur Vadisi (R15). Ir.-Tur.

\section{Iris galatica Siehe/Kaba navruz}

Ovacık, $48 \mathrm{~km}$ from Tunceli to Ovacık (Munzur Valley), 1200 m, steppe, 11.iv.2015, Armağan 6385

(MA). Ir.-Tur., End.

\section{Iris persica L./Buzala}

Tunceli-Ovacık arası, Dilek Köyü girişi, 12.iv.2012, E.Yüce 2007, 2008 (R13); Tunceli-Ovacık arası, 15. km, 12.iv.2012, E.Yüce 2012 (R13); TunceliOvacık arası, Munzur Vadisi Milli Parkı çıkışı, 1215 m, 20.iv.2012, E.Yüce 2040 (R13); Tunceli-Ovacık arası, Anafatma civarı, 940 m, 06.iii.2013, E.Yüce 2454 (R13); Tunceli-Ovacık arası, Munzur Vadisi Milli Parkı çıkışı, 1215 m, 09.iv.2013, E.Yüce 2488, 2489 (R13); Munzur Vadisi (R15). Ir.-Tur.

\section{Iris reticulata M.Bieb. var. reticulata/ Karakörpeze}

Ovacık, $48 \mathrm{~km}$ from Tunceli to Ovacık (Munzur Valley), 1200 m, steppe, 11.iv.2015, Armağan 6383 (MA); Tunceli-Ovacık arası, Munzur Vadisi Milli Parkı çıkışı, 1215 m, 20.iv.2012, E.Yüce 2032 (R13); Tunceli-Ovacık arası, Munzur Vadisi Milli Parkı çıkışı, 1215 m, 09.iv.2013, E.Yüce 2490 (R13); Ovacık, Akyayık Köyü, 1315 m, 22.iv.2013, E.Yüce 2579 (R13); Ovacık, Güneykonak Köyü, 1230 m, 14.iv.2013, E.Yüce 2642, 2650 (R13); Munzur Vadisi (R15). Ir.-Tur. 


\section{Iris sari Schott ex Baker/Anakurtkulağı}

Ovacık, $3 \mathrm{~km} \mathrm{~N}$ of Işıkvuran, Munzur Mountains, 2050 m, mountain steppe, 04.vi.2014, Armağan 4240 (MA); Pülümür, foots of Silbüs Dağı, clearing of Quercus petraea subsp. pinnatiloba forest, 1600-2000 m, Yıldırımlı 3367 (R4); Ovacık (Merkez), Yeşilyazı, 1550 m, 21.v.2012, E.Yüce 2091 (R13); Ovacık, Akyayık Köyü, 1315 m, 12.v.2013, E.Yüce 2601 (R13); Tunceli-Ovacık arası, Munzur Vadisi Milli Parkı çıkışı, 1215 m, 14.v.2013, E.Yüce 2609 (R13); Tunceli-Ovacık arası, Munzur Vadisi Milli Parkı çıkışı, 1215 m, 24.v.2013, E.Yüce 2810 (R13); Munzur Vadisi (R15). Ir.-Tur., End.

\section{IXIOLIRIACEAE}

Ixiolirion tataricum (Pall.) Schult. \& Schult.f. var. tataricum (= Ixiolirion tataricum (Pall.) Schult. \& Schult.f. subsp. montanum (Labill.) Takht.) /

\section{Köpekotu}

Tunceli center, $16 \mathrm{~km}$ from Tunceli to Pülümür (Pülümür Valley), oak openings, $1060 \mathrm{~m}$, 01.v.2015, Armağan 6683 (MA); Pülümür, 2.5 km from Kocatepe to Sarıgül, near to Sarıül crossroad, 1970 m, steppe, 05.vi.2014, Armağan 4343 (MA); Pülümür, from Kırmızıköprü to Mezra Köyü, 1400 m, 21.v.1981, Yıldırımlı 4373 (R4); TunceliOvacık arası, Anafatma civarı, 940 m, 08.v.2012, E.Yüce 2063 (R13); Tunceli-Ovacık arası, Avsugar Köprüsü çevresi, 995 m, 29.v.2012, E.Yüce 2144 (R13); Tunceli-Ovacık arası, Anafatma civarı, 940 m, 27.iv.2013, E.Yüce 2541 (R13); Tunceli-Ovacık arası, Dedeağaç Köyü, 1380 m, 14.v.2013, E.Yüce 2603 (R13); Tunceli-Ovacık arası, Işıkvuran Köyü çıkışı, 26.v.2013, E.Yüce \& i.Eker 2677 (R13); Tunceli center, c. $14.1 \mathrm{~km}$ NE of Tunceli, road D882 to Gökçek, 2.2 km SSW Gökçek, locality called Kutudere, $1000 \mathrm{~m}$, dry meadows and oak forest, 18.v.2011, V.E. 11-0095 (R14); Tunceli center, c. 7.4 km ENE of Tunceli, road D882 to Gökçek, locality called Marçik 940 m, former field and surrounding meadows and shrubland, 18.v.2011, V.E. 11-0077 (R14); Tunceli center C. $14.1 \mathrm{~km}$ NE of Tunceli, road D882 to Gökçek, 2.2 km SSW Gökçek, locality called Kutudere, $1000 \mathrm{~m}$, dry meadows and oak forest, 18.v.2011, V. \& E. 11-0099 (R14); Munzur Vadisi (R15). Ir.-Tur.

\section{LILIACEAE}

\section{Fritillaria alburyana Rix/Pembelâle}

Ovacık, $3 \mathrm{~km} \mathrm{~N}$ of Işıkvuran, Munzur Mountains, 2480 m, rocky areas, 05.vi.2015, Armağan 6557

(MA); Ovacık, Karagöl vadisi, Munzur dağları, 13001500 m, 06.v.1979, Yıldırımlı 1373 (R4); Ovacık, Munzur Dağları, around Kankala, above Karataş Köyü, 1800-2000 m, 08.v.1979, Yıldırımlı 1490 (R4); Ovacık, Mercan Vadisi, 19.v.2013, E.Yüce 2760 (R13); Munzur Vadisi (R15). Ir.-Tur., End.

Fritillaria crassifolia Boiss. \& A.Huet subsp. crassifolia/Boynubükük

Tunceli center, $19 \mathrm{~km}$ from Tunceli to Çıralı, 1870 m, steppe scree, 01.v.2015, Armağan 6686 (MA); Ovacık, Karagöl vadisi, Munzur dağları, 13001500 m, 06.v.1979, Yıldırımlı 1393 (R4); Ovacık, Güneykonak Köyü, 1227 m, 08.iv.2013, E.Yüce 2501 (R13); Ovacık, Güneykonak Köyü, 1227 m, 13.iv.2013, E.Yüce 2648 (R13); Munzur Vadisi (R15). Ir.-Tur., End.

\section{Fritillaria imperialis L./Ağlayangelin}

Ovacık, 7 km from Yoncalı to Ağaçpınar, 2015 m, rocky hillside, 01.vi.2015, Armağan 6538 (MA); Ovacık, 3 km N of Işıkvuran, Munzur Mountains, 2020 m, rocky areas, 04.vi.2014, Armağan 4251 (MA); Tunceli-Ovacık arası, Işıkvuran Köyü çıkışı, 26.v.2013, E.Yüce \& I..Eker 2678 (R13); Munzur Vadisi (R15). Ir.-Tur.

Fritillaria pinardii Boiss. (=Fritillaria armena Boiss.)/Mahçuplâle

Ovacık, $7 \mathrm{~km}$ from Yoncalı to Ağaçpınar, $2015 \mathrm{~m}$, pastures, 01.vi.2015, Armağan 6542 (MA); Ovacık, Munzur Dağları, around Kankala, above Karataş Köyü, 1800-2000 m, 08.v.1979, Yıldırımlı 1483 (R4); Tunceli-Ovacık arası, 45. km, 08.v.2012, E.Yüce 2044 (R13); Ovacık, Havaçor Vadisi girişi, dağ yamacı, 08.v.2012, E.Yüce 2069 (R13); Tunceli-Ovacık arası, Venk Köprüsü çevresi, 1040 m, 14.v.2013, E.Yüce 2608 (R13); Tunceli-Ovacık arası, Güneykonak Köyü, 1230 m, 11.iv.2013, E.Yüce 2643 (R13); Ovacık, Köseler Köyü, 27.iv.2013, E.Yüce 2626 (R13); Munzur Vadisi (R15). Ir.-Tur. 


\section{Fritillaria whittallii Baker/Çam Ialesi}

Ovacık, Karagöl vadisi, Munzur dağları, 1400 m, 05.v.1979, Yıldırımlı 1333 (R4); Munzur Vadisi (R15). E.Medit., End.

\section{Gagea bulbifera (Pall.) Salisb./Düğmeli yıldız}

Tunceli center, $15 \mathrm{~km}$ from Tunceli to Ovacık (Munzur Valley), 995 m, steppe, 11.iv.2015, Armağan 6401 (MA). Euro.-Sib.

Gagea bohemica (Zauschn.) Schult. \& Schult.f./ Sarıyıldız

Nazımiye, $31 \mathrm{~km}$ from Tunceli to Pülümür (Pülümür Valley), 1050 m, steppe, 10.ii.2015, Armağan 6368 (MA); Tunceli-Ovacık arası, Anafatma civarı, 940 m, 30.iii.2013, E.Yüce 2474 (R13).

\section{Gagea chanae Grossh./Çan yıldızı}

Tunceli center, $15 \mathrm{~km}$ from Tunceli to Ovacık (Munzur Valley), 995 m, steppe, 11.iv.2015, Armağan 6401 (MA); Ovacık, Karagöl vadisi, Munzur dağları, 1400 m, 05.v.1979, Yıldırımlı 1328 (R4); Tunceli-Ovacık arası, 30. km, 08.v.2012, E.Yüce 2065 (R13); Munzur Vadisi (R15). Ir.-Tur.

\section{Gagea gageoides (Zucc.) Vved./Tokalıyıldız}

Nazımiye, 12 km NE of Büyükyurt, (Dereova town, 5 km SE of Aşağımarkosör), 1926 m, 01.vi.2015, Armağan 6527 (MA); Pülümür, 11 km NW of Ardıçlı (Gersunut), Munzur Mountains, 2510 m, rocky areas, 18.vi.2014, Armağan 4873 (MA); Ovacık, 3 km N of Işıkvuran, Munzur Mountains, 2080 m, rocky crevices, 04.vi.2014, Armağan 4257 (MA); Nazımiye, Tunceli-Erzincan yolu, Büyükyurt Köyü, 1745 m, 21.v.2011, E.Vitek, C.Ergin 11-0174 (R13); Nazımiye, side valley of road D882 Tunceli - Erzincan, upper part of village Büyükyurt 1745 m, at fence, 21.v.2011, V.E. 11-0174 (R14). Ir.-Tur.

\section{Gagea glacialis K. Koch/Buzyıldızı}

Nazımiye, Dereova town, $5 \mathrm{~km} \mathrm{SE}$ of Aşağımarkosör, $2144 \mathrm{~m}$, near to melted snow in slopes, 01.vi.2015, Armağan 6524 (MA); Munzur Da. above Ovacık, turf by melting snow, c. 2800 m, 17.vii.1957, Davis 31286 \& Hedge (R6); Tunceli-Ovacık arası, Munzur Vadisi Milli Parkı çıkışı, 1215 m, 09.iv.2013, E.Yüce 2493 (R13); Ovacık, Güneykonak Köyü, 1230 m, 08.iv.2013, E.Yüce 2497 (R13). Ir.-Tur.
Gagea liotardii (Sternb.) Schult. \& Schult.f. (=Gagea fragifera (Vill.) E.Bayer \& G.López, = Gagea fistulosa (Ramond ex DC) Ker-Gawl.)/ Yayla yıldızı

Tunceli center, $7 \mathrm{~km}$ from Tunceli to Pülümür (Pülümür Valley), $934 \mathrm{~m}$, steppe, 12.iv.2015, Armağan 6425 (MA).

\section{Gagea luteoides Stapf/Altınyıldız}

Ovacık, $8 \mathrm{~km}$ from Ovacık to Tunceli, $500 \mathrm{~m} \mathrm{~N}$ of Yaylagünü, $1212 \mathrm{~m}$, pastures in stream side, 11.iv.2015, Armağan 6407 (MA); Munzur Da. above Ovacık, around Kankala, above Karataş Köyü, 1800-2000 m, 08.v.1979, Yıldırımlı 1494 (R6); Tunceli-Ovacık arası, Anafatma civarı, 940 m, 20.iv.2012, E.Yüce 2019 (R13).

\section{Gagea minima (L.) Ker Gawl./Minik yıldız}

Munzur vadisi (R15). Ir.-Tur.

\section{Tulipa armena Boiss. var. armena/Dağlâlesi}

Ovacık, $7 \mathrm{~km}$ from Yoncalı to Ağaçpınar, 2015 m, steppe, 01.vi.2015, Armağan 6543 (MA); Ovacık, Munzur Dağları, around Kankala, above Karataş Köyü, 1800-2000 m, 08.v.1979, Yıldırımlı 1495 (R4); Tunceli-Ovacık arası, 45. km, 08.v.2012, E.Yüce 2046 (R13); Ovacık, Munzur gözeleri, 1300 m, 08.v.2012, E.Yüce 2075 (R13); TunceliOvacık arası, Venk Köprüsü çevresi, 1040 m, 08.v.2012, E.Yüce 2079 (R13); Ovacık (Merkez), Yeşilyazı, 1275 m, 21.v.2012, E.Yüce 2089 (R13); Ovacık, Akyayık Köyü, 1315 m, 22.iv.2013, E.Yüce 2565a (R13); Ovacık, Güneykonak Köyü, 1230 m, 08.v.2013, E.Yüce 2653 (R13); Ovacık, c. 18 km NE of Ovacik, NE of small river dam, locality called Şahverdi, 1505 m, 17.v.2011, V.E. 11-0040 (R14); Munzur Vadisi (R15). Ir.-Tur.

Tulipa armena Boiss. var. galatica (Freyn) Eker/ Dağlâlesi

Ovacık, $7 \mathrm{~km}$ from Yoncalı to Ağaçpınar, $2015 \mathrm{~m}$, steppe, 01.vi.2015, Armağan 6695 (MA); Ovacık, Akyayık Köyü, 1315 m, 22.iv.2013, E.Yüce 2565b (R13); Munzur Vadisi (R15). Ir.-Tur.

\section{Tulipa julia K.Koch/Yabanlâlesi}

Tunceli center, $26 \mathrm{~km}$ from Tunceli to Ovacık (Munzur Valley), 1060 m, steppe, 26.v.2014, 
Armağan 4029 (MA); Munzur Da. above Ovacık, 1800 m, D. 31882 (R6); Ovacık, Akyayık Köyü, 1315 m, 22.iv.2013, E.Yüce 2582; Ovacık, Güneykonak Köyü, 1230 m, 24.iv.2013, E.Yüce 2638 (R13). Ir.Tur.

\section{ORCHIDACEAE}

\section{Anacamptis pyramidalis (L.) Rich./Sivrisalep}

Ovacık, N of Eskigedik, Munzur Mountains, 2160 m, oak openings, 19.vi.2014, Armağan 4950 (MA); Mazgirt, road of Elazı̆̆ - Tunceli, between Güneşdere and Kızılcık, 1090 m, oak openings, 02.vi.2014, Armağan 4113 (MA); Tunceli center, road of Tunceli - Elazığ, $700 \mathrm{~m}$ after from Aktuluk, 930 m, oak openings, 22.v.2015, Armağan 3690 (MA); Elazığ to Tunceli, 900 m, Taub. 78.277 (R6); Tunceli-Ovacık arası, Aşağıtorunoba, 09.vi.2013, E.Yüce 2994 (R13); Ovacık, 27.5 km NNW Tunceli, road Aşağıtorunoba-Agdat, $1825 \mathrm{~m}$, semihumid meadow, 18.vi.2013, V.Y.E. \& M. 13-0373 (R14).

\section{Cephalanthera damasonium (Mill.) Druce/ Ormankuşçuğu}

Ovacık, Güneykonak Köyü, 1230 m, 08.v.2013, E.Yüce 2652 (R13). Euro.-Sib.

Cephalanthera epipactoides Fisch. \& C.A.Mey./ Ana çamçiçeği

Tunceli center, c. $8.1 \mathrm{~km}$ ESE of Tunceli, road to Ambar, $970 \mathrm{~m}$, roadside in oak forest, 19.v.2011, V.E. 11-0105 (R14). E.Medit.

Cephalanthera kotschyana Renz \& Taubenheim/ Koç salebi

c. $3 \mathrm{~km} \mathrm{~N}$. of Pülümür pass, $1600 \mathrm{~m}$, Baumann 5550 (R6). End.

Cephalanthera kurdica Bornm. ex Kraezl./ Kurtkuşçuğu

Tunceli center, $17 \mathrm{~km}$ from Tunceli to Pülümür (Pülümür Valley), $986 \mathrm{~m}$, moist shaded places, 29.v.2015, Armağan 6460 (MA); Tunceli center, road of Tunceli - Sütlüce, $2 \mathrm{~km}$ after from junction, 1050 m, oak openings, 23.v.2015, Armağan 3806 (MA); Ovacık, Akyayık Köyü, 1315 m, 10.vi.2013, E.Yüce 2791 (R13). Ir.-Tur.

\section{Cephalanthera longifolia (L.) Fritsch / Kuğusalebi}

Tunceli center, $12 \mathrm{~km}$ from Geyiksuyu to Eğriyamaç, 1575 m, under the forest, 23.v.2015, Armağan 6682 (MA); Tunceli, Kalan river valley, $1 \mathrm{~km}$ from Sarıtaş to Gözen (iksor), $1160 \mathrm{~m}$, under the forest, 30.v.2015, Armağan 6504 (MA); Ovacık, Akyayık Köyü, 1315 m, 13.v.2013, E.Yüce 2602 (R13). Euro.Sib.

Cephalanthera x taubenheimii H.Baumann/ Melez ormankuşçuğu

Inter Tunceli et Erzincan, 8 km N. Pülümür Geçidi, 1600 m, 30.v.1981, H. Baumann (R6). End.

Dactylorhiza iberica (M.Bieb. ex Willd.) Soó/ Kırım salebi

Pülümür, road of Pülümür - Yedisu, 6 km after from Derindere, 1960 m, moist meadows, 22.vii.2014, Armağan 5551 (MA); Pülümür, between Bardakçı and Derindere, $1980 \mathrm{~m}$, moist meadows, 22.vii.2014, Armağan 5512 (MA); above Pülümür, 1900 m, D. 31594 (R6); Ovacık, banks of Munzur Suyu, 1200 m, 18.vi.1979, Yıldırımlı 2037 (R4); Ovacık, Akyayık Köyü, 1315 m, 10.vi.2013, E.Yüce 2792 (R13); Ovacık, Güneykonak Köyü, 1230 m, 05.vi.2013, E.Yüce 2804 (R13); Tunceli center, c. $14.1 \mathrm{~km}$ NE of Tunceli, road D882 to Gökçek, 2.2 km SSW Gökçek, locality called Kutudere, 1000 m, dry meadows and oak forest, 18.v.2011, V.E. 110097 (R14); Munzur Vadisi (R15). E.Medit.

Dactylorhiza osmanica (Klinge) P.F.Hunt \& Summerh. var. anatolica (Nelson) Renz \& Taubenheim/Osmanlısalebi

Pülümür pass, 1900 m, Renz \& Taub. 78.452 (R6). End.

Dactylorhiza osmanica (Klinge) P.F.Hunt \& Summerh. var. osmanica/Osmanlısalebi

Ovacık, 8 km from Eskigedik to Işıkvuran, 2120 m, 19.vi.2014, Armağan 4925 (MA); Ovacık, 6 km from Işıkvuran to Eskigedik, 2250 m, meadows, 19.vi.2014, Armağan 4921 (MA); Ovacık, 48 km from Tunceli to Ovacık (Munzur Valley), 1190, meadows, 26.v.2014, Armağan 4059 (MA); Ovacık, Karagöl vadisi, Munzur dağları, 1400 m, 05.v.1979, Yıldırımlı 1331 (R4); Pülümür, by stream, 
1500 m, 15.vi.1980, Yıldırımlı 3253 (R4); Ovacık, Mercan Vadisi, 29.v.2012, E.Yüce 2119 (R13); Ovacık, Güneykonak Köyü, 1230 m, 08.v.2013, E.Yüce 2635 (R13); Tunceli-Ovacık yolu, ÖrenönüYakatarla Köyü arası, 26.v.2013, E.Yüce \& i.Eker 2668 (R13); Ovacık, road Ovacık - Hozat, c. 6.4 km SSSW Ovacık, $1665 \mathrm{~m}$, humid place in oak forest 12.vi.2013, V.Y.E. \& M. 13-0046 (R14); Ovacık, 27.5 km N Tunceli, road Aşağıtorunoba - Agdat, 1860 m, stony slope, limestone, 18.vi.2013, V.Y.E. \& M. 130399b (R14); Ovacık, c. 14 km WWSW Ovacık, 0.35 $\mathrm{km}$ W Ziyaret, near the fountains of river Munzur, 1320 m, near river, 12.vi.2013, V.Y.E. \& M. 13-0038 (R14). Ir.-Tur., End.

Dactylorhiza romana (Seb.) Soo subsp. georgica (Klinge) Soó ex Renz \& Taubenheim/Çamkökü

Ovacık, $7 \mathrm{~km}$ from Yoncalı to Ağaçpınar, $2165 \mathrm{~m}$, meadows, 01.vi.2015, Armağan 6536 (MA).

Dactylorhiza romana (Sebast.) Soó subsp. romana/Elçik

Tunceli center, road of Tunceli - Çıralı, $12 \mathrm{~km}$ after from Tunceli - Sütlüce crossroad (13 km to Çıralı), 1760 m, meadows, 01.vi.2015, Armağan 6515 (MA); Ovacık, along Mercan Çayı, 1100-1200 m, 07.v.1979, Yıldırımlı 1429 (R6); Ovacık, Güneykonak Köyü, 1230 m, 08.v.2013, E.Yüce 2656a (R13). Medit.

Dactylorhiza umbrosa (Karelin \& Kirilow) Nevski var. umbrosa/Gövdeli salep

Pülümür, 14 km from Pülümür to Tunceli (Pülümür Valley), 1232 m, moist meadows, 29.v.2015, Armağan 6485 (MA); Ovacık, Işıkvuran village, 1710 m, meadows, 04.vi.2014, Armağan 4291 (MA). Ir.-Tur.

\section{Epipactis palustris (L.) Crantz/Danakıranotu}

Above Pülümür, 1900 m, D. 31595 (R6).

Limodorum abortivum (L.) Sw. var. abortivum/ Saçuzatan

10 km W. of Geyiksu, 1450 m, Taub. 81.148 (R6).

\section{Ophrys apifera Huds./Arısalebi}

Tunceli-Hozat arası, Büyükçeşme, 01.vi.2012, E.Yüce 2317 (R13); Tunceli-Pülümür yolu, Kutik Şelalesi, 04.vi.2012, E.Yüce 2374 (R13).
Ophrys oestrifera M.Bieb. subsp. oestrifera/ Sineksalebi

Tunceli center, $17 \mathrm{~km}$ from Tunceli to Pülümür (Pülümür Valley), 986 m, meadows, 29.v.2015, Armağan 6459 (MA); Pülümür, 700 m W of Kırmızı bridge, 1370 m, meadows, 05.vi.2014, Armağan 4318 (MA); 3 km E. of Ovacık, 1150 m, Taub. 81.139 (R6); Tunceli-Ovacık arası, Halbori Gözeleri, 26.v.2013, E.Yüce 2665 (R13).

Ophrys phrygia H.Fleischm. \& Bornm./Yunus salebi

Nazımiye, $6.5 \mathrm{~km}$ from Nazımiye to Tunceli, $400 \mathrm{~m}$ after from crossroad of Geriş, 1340 m, meadows, 03.vi.2014, Armağan 4161 (MA); c. 8 km S. of Tunceli, 850 m, Taub. \& Mangold 81.78 (R6).

Ophrys reinholdii Spruner ex Fleischm. subsp. straussii (H.Fleischm.) E.Nelson / Sidikli salep

Nazımiye, 4 km NE of Büyükyurt, 1710 m, under the forest, 01.vi.2015, Armağan 6521 (MA); Tunceli to Pülümür 1150 m, Taub. 78.280 (R6).

Orchis coriophora L. subsp. coriophora/ Pirinççiçeği

Pülümür, $6 \mathrm{~km}$ from Kocatepe to Çakırkaya, 1510 m, humid places, 05.vi.2014, Armağan 4330 (MA); Ovacık, 4 km from Aşağıtorunoba to Ağaçpınar, 1800 m, moist meadows, 04.vi.2014, Armağan 4311 (MA); Ovacık, $15 \mathrm{~km}$ from road of Ovacık - Tunceli to Işıkvuran, $1670 \mathrm{~m}$, moist meadows, 04.vi.2014, Armağan 4236 (MA); Ovacık, 48 km from Tunceli to Ovacık (Munzur Valley), $1190 \mathrm{~m}$, moist meadows, 26.v.2014, Armağan 4060 (MA); Tunceli center, 5 $\mathrm{km}$ from Tunceli to Ovacık (Munzur Valley), 940 m, meadows in stream side, 25.v.2014, Armağan 3950 (MA); Ovacık, banks of Munzur Suyu, 1200 m, 18.vi.1979, Yıldırımlı 2035 (R4); Tunceli-Ovacık arası, Halbori Gözeleri, 26.v.2013, E.Yüce \& i.Eker 2663 (R13); Tunceli-Hozat arası, 20. km, 27.v.2013, E.Yüce \& İ.Eker 2694 (R13); Munzur Vadisi (R15).

Orchis laxiflora Lam. subsp. dielsiana Soó/ Horanta salebi

Pülümür, 14 km from Pülümür to Tunceli (Pülümür Valley), 1232 m, humid places, 29.v.2015, Armağan 6484 (MA). 
Orchis laxiflora Lam. subsp. laxiflora/ Salepsümbülü

Ovacık, $48 \mathrm{~km}$ from Tunceli to Ovacık (Munzur Valley), $1190 \mathrm{~m}$, moist meadows, 26.v.2014, Armağan 4062 (MA); Tunceli-Hozat arası, 20. km, 27.v.2013, E.Yüce \& i.Eker 2692 (R13).

Orchis mascula (L.) L. subsp. mascula/Ersalebi

Tunceli-Ovacık arası, Işıkvuran Köyü, 18.v.2013, E.Yüce 2812a (R13).

Orchis mascula (L.) L. subsp. pinetorum (Boiss. \& Kotschy) G.Camus/Çam salebi

Pülümür, $2.5 \mathrm{~km}$ from Kocatepe to Sarıgül, near to Sarıgül's crossroad, 1970 m, oak openings, 05.vi.2014, Armağan 4357 (MA); c. $10 \mathrm{~km} \mathrm{S.} \mathrm{of}$ Tunceli, 1050 m, Taub. 78.278 (R6).

Orchis militaris L. subsp. stevenii (Rchb.f.) B.Baumann, R.Lorenz \& Ruedi Peter/Gürbüzsalep

$3 \mathrm{~km}$ E. of Ovacık, $1150 \mathrm{~m}$, Taub. 81.135 (R6); Pülümür, Kırmızıköprü, Salördek Köyü, 19.v.2013, E.Yüce 2712 (R13). Hyr.-Eux.

\section{Orchis pallens L./Solgunsalep}

Ovacık, Akyayık Köyü, 1315 m, 04.v.2013, E.Yüce 2578 (R13). Euro.-Sib.

Orchis palustris Jacq. subsp. palustris/ Çayırsalebi

Tunceli center, $5 \mathrm{~km}$ from Tunceli to Sütlüce, 1085 m, steppe, 12.v.2015, Armağan 6680 (MA); c. 48 km from Tunceli to Pülümür, 1300 m, D. 29246 (R6); Ovacık, banks of Munzur Suyu, 1200 m, 18.6.1979, Yıldırımlı 2036 (R4); Pülümür, foots of Kuzdere Tepesi, steppe, 1500-1700 m, 14.6.1980, Yıldırımlı 3077 (R4); Ovacık, Akyayık Köyü, 1315 m, 23.iv.2013, E.Yüce 2580 (R13); Munzur Vadisi (R15).

\section{Orchis punctulata Steven ex Lindl./Selef}

Tunceli center, $3 \mathrm{~km}$ from Tunceli to Ovacık (Munzur Valley), 920 m, steppe, 01.v.2015, Armağan 6685 (MA); Pülümür, N of Turnadere, 1424 m, dried steppe hills, 29.v.2015, Armağan 6492 (MA); Erdige to Çemişgezek, Attila ISTF 16629 (R6);
Pülümür, Kırmızıköprü, Salördek Köyü, 19.v.2013, E.Yüce 2707 (R13). E.Medit.

\section{Orchis sancta L./Püren salebi}

Ovacık, 27.5 km NNW Tunceli, road Aşağıtorunoba - Agdat, 1825 m, semihumid meadow, 18.vi.2013, V.Y.E. \& M. 13-0372 (R14).

\section{Orchis simia Lam./Salep püskülü}

Tunceli center, $3 \mathrm{~km}$ from Tunceli to Ovacık (Munzur Valley), 920 m, steppe, 01.v.2015, Armağan 6689 (MA). Medit.

Orchis spitzelii Sauter ex W.D.J.Koch/Dağ salebi

Tunceli center, road of Tunceli - Çıralı, $12 \mathrm{~km}$ from Tunceli - Sütlüce crossroad (13 km to Çıralı), 1760 m, steppe, 30.v.2015, Armağan 6514 (MA); Tunceli center, road of Tunceli - Çıralı, $15 \mathrm{~km}$ from crossroad of Tunceli - Sütlüce (10 km to Çıralı), 1850 m, steppe, 23.v.2014, Armağan 3829 (MA). Medit.

\section{Orchis tridentata Scop./Katranalacası}

Tunceli center, $16 \mathrm{~km}$ from Tunceli to Pülümür (Pülümür Valley), 1060 m, oak openings, 02.v.2015, Armağan 6684 (MA); Tunceli center, road of Tunceli - Çıralı, $12 \mathrm{~km}$ from Tunceli - Sütlüce crossroad (13 km to Çıralı), $1760 \mathrm{~m}$, meadows, 30.v.2015, Armağan 6513 (MA); Tunceli-Ovacık arası, Anafatma Ziyareti, 940 m, 03.v.2013, E.Yüce 2548 (R13). Medit.

\section{Platanthera chlorantha (Cruster) Rchb./ Çarpıksalep}

Ovacık, Akyayık Köyü, 1315m, 10.vi.2013, E.Yüce 2799 (R13); Ovacık, Güneykonak Köyü, 1230 m, 05.vi.2013, E.Yüce 2806 (R13).

\section{XANTHORRHOEACEAE}

Asphodeline damascena (Boiss.) Baker subsp. damascena/Çekiçlik

Ovacık, Ovacık'ın 9.2 km kuzeydoğusu, 1400 m, 17.v.2011, E.Vitek, C.Ergin 11-0051 (R13). Ir.-Tur.

Asphodeline tenuior (Fisch. ex M.Bieb.) Ledeb. subsp. tenuiflora (K.Koch) Tuzlaci var. puberulenta 


\section{Tuzlaci/Tesbihçik}

Pülümür, 3 km from Çakırkaya to Dereboyu, 1830 m, steppe, 05.vi.2014, Armağan 4390 (MA); d. Ovacık, nr Köseköyü, 1200 m, Yıldırımlı 1370 (R6); Pülümür, E.S. Brown 2434 (R6); 11 km from Tunceli to Pülümür, 1350 m, Tuzlacı (R6); Ovacık, below Köse köyü, Karagöl Vadisi, 1200 m, 05.v.1979, Yıldırımlı 1370 (R4); Ovacık, Akyayık Köyü, 1315 m, 22.iv.2013, E.Yüce 2571 (R13); Ovacık, Akyayık Köyü girişi, tarla içi, 27.v.2013, E.Yüce \& i.Eker 2703 (R13). Ir.-Tur., End.

Asphodeline tenuior (Fisch. ex M.Bieb.) Ledeb. subsp. tenuiflora (K.Koch) Tuzlacı var. tenuiflora/ Tesbihçik

Mazgirt, Akpazar town, S of Yenice ( $\mathrm{N}$ of Şevki mezrası), 1250 m, steppe, 02.vi.2014, Armağan 4122 (MA); Nazımiye, road Nazımiye - Dereova, 3.9 km SE Dereova 1415 m, dry gravel, 15.vi.2013, V.Y.E. \& M. 13-0222 (R14). Ir.-Tur.

\section{Eremurus cappadocicus J.Gay ex Baker/Mor çiriş}

Mazgirt, road of Elazığ - Tunceli, between Güneşdere and Kızılcık, 1090 m, steppe, 02.vi.2014, Armağan 4118 (MA). Ir.-Tur.

\section{Eremurus spectabilis M.Bieb./Çiriş}

Tunceli center, $18 \mathrm{~km}$ from Tunceli to Ovacık (Munzur Valley), 990 m, steppe, 30.v.2015, Armağan 6511 (MA); Ovacık, 3 km N of Işıkvuran, Munzur Mountains, $2050 \mathrm{~m}$, mounatin steppe, 04.vi.2014, Armağan 4241 (MA); Tunceli center, road of Tunceli - Çıralı, $16 \mathrm{~km}$ from crossroad of Tunceli - Sütlüce (9 km to Çıralı), 1850 m, steppe, 23.v.2014, Armağan 3832 (MA); Pülümür, foots of Silbüs Dağı, clearing of Quercus petraea subsp. pinnatiloba forest, 1600-2000 m, Yıldırımlı 3351 (R4); Ovacık, Karagöl Vadisi, from Dılap Yaylası to Koyungölü Köyü by path, 1250-2250 m, 08.vii.1980, Yıldırımlı 3608 (R4); Tunceli-Ovacık arası, 40. km, 21.v.2012, E.Yüce 2087 (R13); Munzur Vadisi (R15). Ir.-Tur.

\section{DISCUSSION}

Accounting for taxa according to floristic regions were as follows; 50\% (74 taxa) Irano - Turanian, 22\% (15 taxa) Mediterranean, 6\% (9 taxa) Euro - Siberian, and 29\% (44 taxa) wide spread and/or unknown regions (Figure 2 ).

The families with the most taxa in the research area were Amaryllidaceae (37 taxa), Orchidaceae (33 taxa), Asparagaceae (18 taxa), and Liliaceae (16

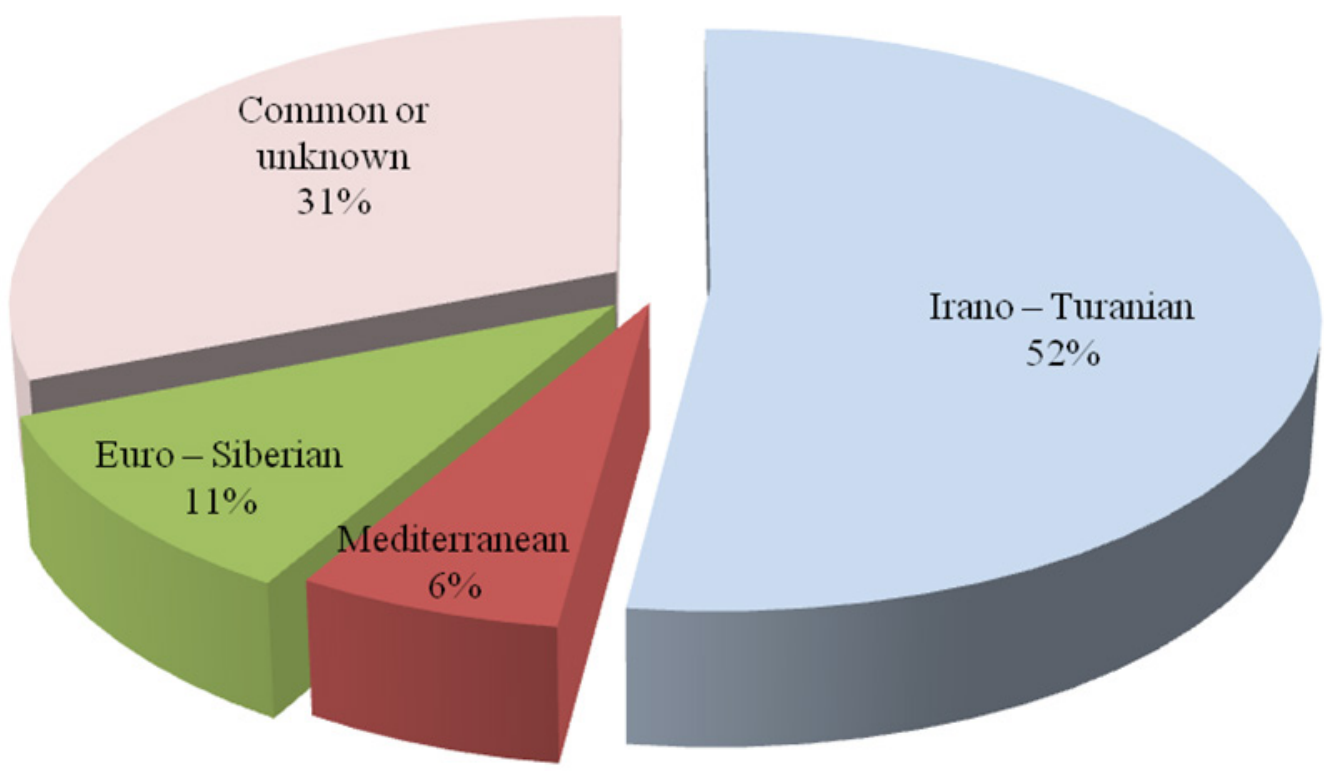

Figure 2. The distribution of taxa depending on phytogeographical regions. 
taxa) (Figure 3, Table 1). The major genera in this study with relation to the number of taxa were Allium L. (36 taxa), Ornithogalum L. (15 taxa), Orchis L. (13 taxa), Gagea Salisb. (8 taxa), Bellevalia Lapeyr. (7 taxa), Iris L. (6 taxa), Muscari Mill. (6 taxa), Dactylor hiza Necker ex Nevski (6 taxa), and Cephalanthera Rich. (6 taxa). Five of 32 genera contain $53 \%$ of all taxa in the research area (Figure 4, Table 2).
In comparison the results of the current study and the previous floristic studies in Tunceli province; 105 taxa in this study, 44 taxa in Reference 4, 41 taxa in Reference 6, 3 taxa in Reference 8, one taxon in Reference 11, one taxon in Reference 12, 91 taxa in Reference 13, 21 taxa in Reference 14, and 49 taxa in Reference 15 were found. As a result, 26 taxa were newly recorded for the province of Tunceli.

Table 1. A comparison of studies in Tunceli in terms of major families.

\begin{tabular}{|c|c|c|c|c|c|c|}
\hline \multirow[t]{2}{*}{ The studies } & \multicolumn{6}{|c|}{ The number of taxa belonging to families } \\
\hline & Amaryllidaceae & Orchidaceae & Asparagaceae & Liliaceae & Iridaceae & Total \\
\hline This study & 28 & 20 & 21 & 14 & 11 & 94 \\
\hline Reference 6 & 11 & 15 & 8 & 3 & 1 & 38 \\
\hline Reference 4 & 7 & 5 & 11 & 7 & 6 & 36 \\
\hline Reference 8 & 1 & 0 & 1 & 0 & 0 & 2 \\
\hline Reference 11 & 0 & 0 & 1 & 0 & 0 & 1 \\
\hline $\begin{array}{l}\text { Reference } \\
12\end{array}$ & 1 & 0 & 0 & 0 & 0 & 1 \\
\hline $\begin{array}{l}\text { Reference } \\
13\end{array}$ & 19 & 18 & 22 & 12 & 9 & 80 \\
\hline $\begin{array}{l}\text { Reference } \\
14\end{array}$ & 3 & 5 & 4 & 2 & 4 & 18 \\
\hline $\begin{array}{l}\text { Reference } \\
15\end{array}$ & 12 & 3 & 9 & 9 & 9 & 42 \\
\hline
\end{tabular}

Table 2. A comparison of studies in Tunceli in terms of first five genera.

\begin{tabular}{|c|c|c|c|c|c|c|}
\hline \multirow[t]{2}{*}{ The studies } & \multicolumn{6}{|c|}{ The number of taxa belonging to families } \\
\hline & Allium & Ornithogalum & Orchis & Gagea & Bellevalia & Total \\
\hline This study & 27 & 10 & 9 & 7 & 3 & 56 \\
\hline Reference 4 & 6 & 2 & 2 & 2 & 2 & 14 \\
\hline Reference 6 & 11 & 3 & 4 & 2 & 2 & 22 \\
\hline Reference 8 & 1 & 0 & 0 & 0 & 1 & 2 \\
\hline Reference 11 & 0 & 1 & 0 & 0 & 0 & 1 \\
\hline Reference 12 & 1 & 0 & 0 & 0 & 0 & 1 \\
\hline Reference 13 & 18 & 9 & 8 & 5 & 6 & 46 \\
\hline Reference 14 & 3 & 3 & 1 & 1 & 0 & 8 \\
\hline Reference 15 & 11 & 3 & 2 & 2 & 2 & 20 \\
\hline
\end{tabular}




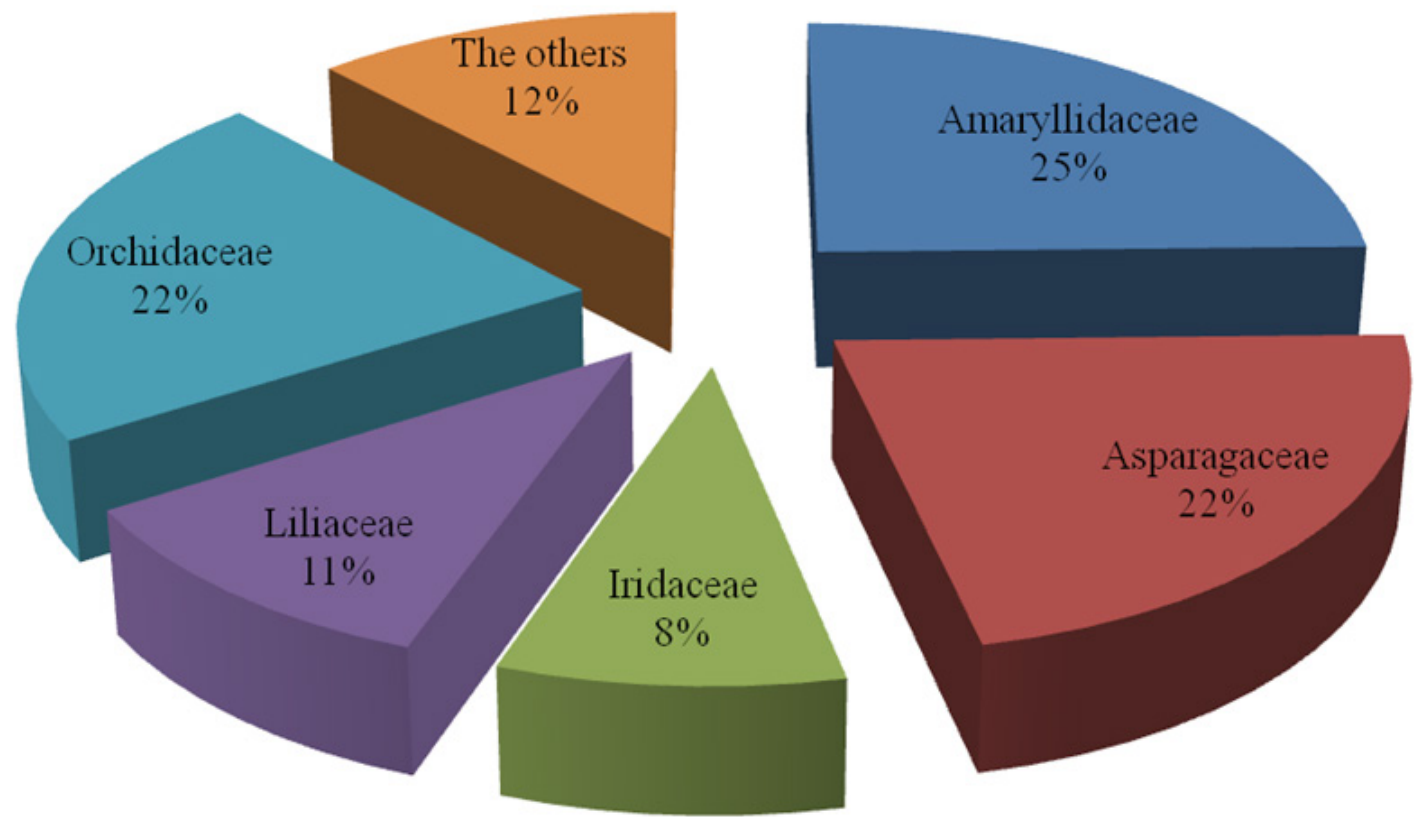

Figure 3. The distribution of families according to number of taxa.

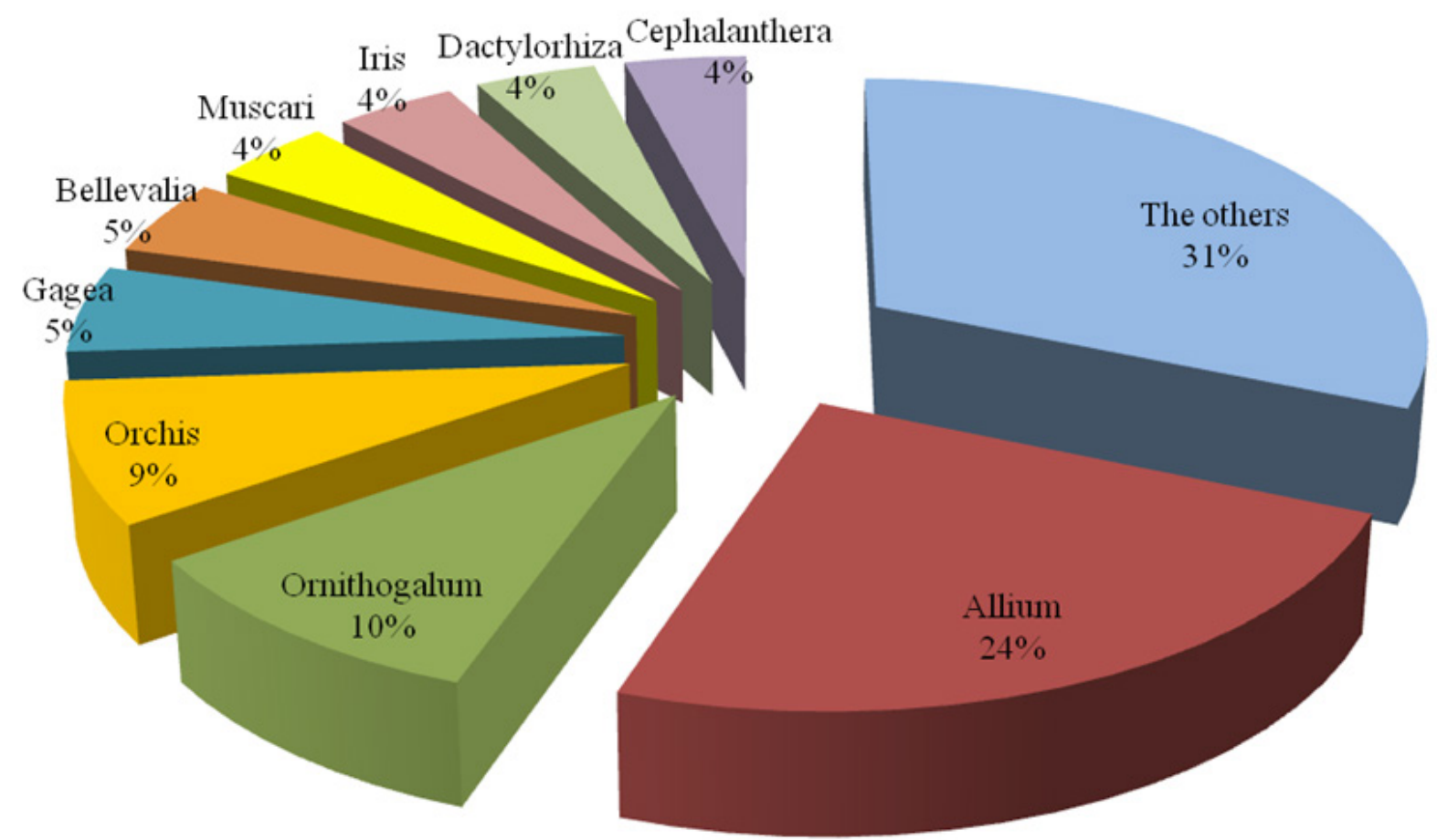

Figure 4. The distribution of genera according to number of taxa. 
Allium cardiostemon Fisch. \& C.A.Mey., Muscari comosum (L.) Mill., Gladiolus atroviolaceus Boiss., Gladiolus italicus Mill., Ixiolirion tataricum (Pall.) Schult. \& Schult.f. var. tataricum, Tulipa armena Boiss. var. armena, Dactylorhiza iberica (M.Bieb. ex Willd.) So, and Dactylorhiza osmanica (Klinge) P.F.Hunt \& Summerh. var. osmanica were the most common taxa in floristic studies carried out in Tunceli.

There are some contradictions between previous research findings, for example Gagea minima (L.) Ker Gawl., given as a new record for the Flora of Turkey from Van in 2015 [16], were determined from the Munzur Valley (Tunceli) by Koyuncu and Aslan in 2009 [15]. Gagea chanae Grossh., given as an interesting finding in previous studies, was already found in Munzur Valley in the researches of Yıldırımlı and Koyuncu \& Aslan $[4,15]$.

The endemism ratio of monocotyledonous petaloids in Tunceli province is $22 \%$ (33 taxa). The ratio of endemism is high because Tunceli has a lot of the microclimatic areas. The climate is so suitable to living of the geophytic plants [13].

The in-situ and ex-situ studies for the preservation of future genetic resources, especielly the endemic species, and also the applied researches such as cultivation of the geophytes for the ornamental plants will be more effective in Tunceli because it has the more suitable and various habitats.

The nature tourism and the transhumance are preferred so much in public of Tunceli. Therefore, the awareness of the public about the recognition and conservation of natural vegetation will contribute to the conservation of nature and the sustainable tourism.

\section{ACKNOWLEDGEMENTS}

This study is based on field studies as part of "Biodiversity Inventory and Monitoring Works on Terrestrial and Inland Water Ecosystems of Tunceli Province" carried out by the Tunceli Office of Turkish Republic of Forestry and Water Affairs, General Directorate of Nature Conservation and National Parks. I would like to thank the aforementioned institution, A.H. Gürsönmez, M. Özel, and Z. Duman.

\section{References}

1. P.H. Davis, Distribution patterns in Anatolia with particular reference to endemism, in: P.H. Davis, P.C. Harper, I.C. Hedge (Eds.), Plant Life of South-West Asia, The Botanical Society of Edinburgh, Edinburgh, UK, 1971.

2. H. Gür, The Anatolian diagonal revisited: Testing the ecological basis of a biogeographic boundary, Zoology in the Middle East, 62 (2016), 189-199.

3. M. Öztürk, A. Tatlı, H. Özçelik, L. Behçet, General Characteristics of Flora and Vegetation Formations of Eastern Anatolia Region and Its Environs (Türkiye), SDU J. Sci., 10 (2015) 23-48.

4. Ş. Yıldırımlı, Flora of Munzur Dağları (ErzincanTunceli), Ot Sis Bot Dergisi 2 (1995) 1-78.

5. S.C. Demir, i. Eker, Petaloid Monocotyledous Flora of Bolu Province, Including Annotations on Critical Petaloid Geophytes of Turkey, PEGEM Akademi, Ankara, Turkey, 2015

6. P.H. Davis, R.R. Mill, K. Tan, Flora of Turkeyandthe East Aegeanlslands, vol. 8, Edinburgh Univ. Press, Edinburgh, UK, 1984

7. P.H. Davis, R.R. Miller, K. Tan, Flora of Turkeyandthe East Aegeanlslands, vol. 10, Edinburgh Univ. Press, Edinburgh, UK, 1988.

8. Güner, N. Özhatay, T. Ekim, K.H.C. Başer, Flora of Turkey and the East Aegean Islands, vol. 11 (supp. 2), Edinburgh Univ. Press, Edinburgh, UK, 2000.

9. Bizim Bitkiler (2018). Version 3.1. Published on the Internet; http://www.bizimbitkiler.org.tr/v3/demo/ details. php? $i d=402 \& t=1$ (accessed: 21 st February).

10. The Plant List, http://www.theplantlist.org, 2013 (accessed: February 2018).

11. N. Özhatay, S. Kültür, Check-List of Additional Taxa to the Supplement Flora of Turkey III, Turk J Bot, 30 (2006) 281-316.

12. N. Özhatay, S. Kültür, B. Gürdal, Check-List of Additional Taxa to the Supplement Flora of Turkey VI, Istanbul Ecz. Fak. Derg., 43 (2013) 33-82.

13. E. Babacan Yüce, İ. Eker, Munzur Vadisi (Tunceli) ve yakın çevresinin geofit florası, Bağ Bahçe Bil. Derg., 4 (2017) 31-49.

14. E.Babacan Yüce, E. Vitek, U. Çakılcıoğlu, Contributions to the Flora of Tunceli (Turkey), International J. Natur. Life Sci., 1 (2017) 39-66.

15. M. Koyuncu, N. Arslan, Munzur Vadisi'nin Biyolojik Çeşitliliğinin Korunması, Ulaşılabilir Yaşam Derneği, Ankara, Turkey, 2009.

16. M. Tekşen, I. Eker, S. Aslan, Gagea minima (L.) Ker Gawl. (Liliaceae): Türkiye için yeni kayıt, Bağbahçe Bil. Derg., 2 (2015) 9-18. 